\title{
Integrating Dynamics into Design and Motion Optimization of a 3-PRR Planar Parallel Manipulator with Discrete Time Transfer Matrix Method
}

\author{
Guoning Si, ${ }^{1}$ Mengqiu Chu, ${ }^{1}$ Zhuo Zhang, ${ }^{2}$ Haijie $\mathrm{Li}^{3}$ and Xuping Zhang ${ }^{3}{ }^{3}$ \\ ${ }^{1}$ University of Shanghai for Science and Technology, School of Medical Instrument and Food Engineering, \\ Shanghai 200093, China \\ ${ }^{2}$ Xi'an University of Posts \& Telecommunications, School of Modern Posts, Xi'an 710061, China \\ ${ }^{3}$ Aarhus University, Department of Engineering, Aarhus 8000, Denmark
}

Correspondence should be addressed to Xuping Zhang; xuzh@eng.au.dk

Received 5 December 2019; Revised 21 March 2020; Accepted 15 April 2020; Published 12 May 2020

Academic Editor: Renato Vidoni

Copyright (c) 2020 Guoning Si et al. This is an open access article distributed under the Creative Commons Attribution License, which permits unrestricted use, distribution, and reproduction in any medium, provided the original work is properly cited.

\begin{abstract}
This paper presents a novel method of dynamic modeling and design optimization integrated with dynamics for parallel robot manipulators. Firstly, a computationally efficient modeling method, the discrete time transfer matrix method (DT-TMM), is proposed to establish the dynamic model of a 3-PRR planar parallel manipulator (PPM) for the first time. The numerical simulations are performed with both the proposed DT-TMM dynamic modeling and the ADAMS modeling. The applicability and effectiveness of DT-TMM in parallel manipulators are verified by comparing the numerical results. Secondly, the design parameters of the 3-PRR parallel manipulator are optimized using the kinematic performance indices, such as global workspace conditioning index (GWCI), global condition index (GCI), and global gradient index (GGI). Finally, a dynamic performance index, namely, driving force index (DFI), is proposed based on the established dynamic model. The described motion trajectory of the moving platform is placed into the optimized workspace and the initial position is determined to finalize the end-effector trajectory of the parallel manipulator by the further optimization with the integrated kinematic and dynamic performance indices. The novelty of this work includes (1) developing a new dynamic model method with high computation efficiency for parallel robot manipulators using DT-TMM and (2) proposing a new dynamic performance index and integrating the dynamic index into the motion and design optimization of parallel robot manipulators.
\end{abstract}

\section{Introduction}

Parallel manipulators with closed-loop mechanical architectures have advantages over serial-link manipulators with open-loop mechanical architectures, such as high precision, large load capacity, fast speed, and high acceleration. Therefore, various types of parallel manipulators have been developed to substitute the serial ones for the precise manipulations at high speed and acceleration. Parallel manipulators have been applied more and more in aerospace [1], precision manufacturing [2], structural engineering [3], surgical robot [4], and other fields.

However, parallel manipulators also have disadvantages compared with serial manipulators. The first primary disadvantage is that parallel manipulators have very complex kinematic equations, which makes dynamic modeling become much more challenging [5]. Compared with the significant efforts and achievements in the kinematics over the past decades, much fewer research efforts were paid toward the dynamics and control of parallel manipulators due to the complicated calculations [6,7]. To promote the applications of parallel manipulators, some fundamental problems must be addressed. One major issue is the computational efficiency, especially for the dynamics and control of parallel manipulators considering the flexibility of their components [8-10].

DT-TMM is a recently developed method for the dynamic modeling of multibody systems, and it is featured with high computation efficiency [11-13]. The DT-TMM 
method has been applied to the dynamic modeling of multibody systems, such as multibarrel rocket [14], selfpropelled gun [15], naval gun [16], and serial manipulators with flexible links [17-19]. One major contribution of this work is to extend the research efforts in the DT-TMM to the dynamic modeling of the 3-PRR planar parallel manipulator and verify its applicability. The proposed methodology in this work can be further extended to the dynamic modeling of other types of planar parallel manipulators and pave the way for the dynamic design and optimization of the mechanical structure.

The second primary disadvantage is a limited workspace [20] and kinematic singularity inside the complete workspace due to the additional constraints imposed by the closed kinematic chains of these mechanisms [21, 22]. Therefore, significant research efforts have been paid to the structure and motion optimization of parallel manipulators using kinematic performance indexes, such as global performance index [23], workspace [24], global stiffness performance [25], and manipulability ellipsoid [26]. To optimize the dynamic design of the planar parallel robot, the maximum driving force is proposed and defined as a dynamic index in this work. By computing the driving force of the parallel manipulator in the process of movement, the maximum driving force required is minimized, when the center position of the moving platform tracks the described trajectory with the desirable kinematic performance.

In this paper, DT-TMM is employed to establish the dynamics model of 3-PRR PPM, and it is verified with the numerical simulations using the virtual work principle and ADAMS. The maximum driving force is adopted as the dynamic performance index for the design and motion optimization of the PPM, combined with Global Workspace Conditioning Index (GWCI), Global Condition Index (GCI), and Global Gradient Index (GGI) [27]. The singleobjective and multiobjective analysis methods are performed to achieve the optimized structure and motion trajectory of the PPM $[28,29]$. Figure 1 is presented to illustrate the methodology for the design and optimization of the 3-PRR planar parallel manipulator by integrated kinematics and dynamics. The twofold contribution of this work is (1) developing a computationally efficient dynamic modeling method for a parallel manipulator by employing DT-TMM and (2) proposing a new dynamic performance index to conduct the dynamic design and motion optimization of parallel robot manipulators with the developed dynamic modeling method. Although the developed dynamic modeling method and optimization strategies are demonstrated with the 3-PRR parallel robot manipulator in this work, it is feasible to extend the developed method and strategies to other types of parallel robot manipulators.

\section{Inverse Kinematics Modeling}

2.1. Configuration and Coordinate System of the Planar Manipulator. The three degrees of freedom of parallel manipulator presented as a case study in this paper is composed of three links, each of which consists of a translational joint and two rotational joints to form a PRR motion structure. Each slider moves along a linear guideway and is driven by a DC motor through the ball screw mechanism. The moving platform and the three sliders are connected by three intermediate links. The two ends of the intermediate links are composed of nondriving revolute joints. The moving platform is an equilateral triangle corresponding to the end-effector, and its motion is determined by the forces transferred from the three intermediate links. The entire moving platform moves along the plane of the $X$-axis and $Y$-axis plane and rotates at an angle around the $Z$-axis of the plane [30].

The rectangular coordinate system $X O Y$ is at the center of the static platform (Figure 2). The origin of each slider is fixed at $A_{i}, i=1,2,3$. The orientation of sliders is at angles $\alpha_{i}$ concerning the base frame XOY, and $\left[\alpha_{1} \alpha_{2} \alpha_{3}\right]=$ $\left[30^{\circ} 150^{\circ} 270^{\circ}\right]$ in this case study. The symbols $\rho_{i}$ represents the distance of the slider from $A_{i} . B_{i}$ is the joint between the slider and the intermediate links. The angles between the intermediate links and the $X$-axis are indicated as $\beta_{i} . C_{i}$ connects the intermediate link and the moving platform. The position of the platform at its mass center $P$ is defined as $\left(X_{p}, Y_{p}\right)$ while $\varphi_{p}$ denotes the orientation angle of the platform and the $X$-axis.

2.2. Inverse Kinematics. The Jacobian matrix of the manipulator is the mapping between the input joint velocity vector and the output velocity vector of the moving platform. The Jacobian matrix of the manipulator can be obtained through inverse kinematics [31]. For the parallel manipulator with three same symmetric kinematic chains, only one of three chains is illustrated for establishing the equation, and the equations of the other two chains can be expressed based on the geometric symmetry (Figure 3).

The close loop geometry equation of each chain can be expressed as

$$
\overrightarrow{A_{i} B_{i}}+\overrightarrow{B_{i} C_{i}}+\overrightarrow{C_{i} P}+\overrightarrow{P O}+\overrightarrow{O A_{i}}=0
$$

The vector in equation (1) is projected onto the $X$ and $Y$ axis:

$$
\left\{\begin{array}{l}
\rho_{i} \cos \alpha_{i}+L_{2} \cos \beta_{i}+L_{3} \cos \gamma_{i}=X_{p}-X_{A_{i}} \\
\rho_{i} \sin \alpha_{i}+L_{2} \sin \beta_{i}+L_{3} \sin \gamma_{i}=Y_{p}-Y_{A_{i}}
\end{array}\right.
$$

where $\left(X_{A_{i}}, Y_{A_{i}}\right)$ are the positions of crossing point $A_{i}$, and $L_{2}$ is the length of the intermediate link, $\alpha_{i}=(4 \pi i-3 \pi / 6)$, $\gamma_{i}=\alpha_{i}+\varphi_{p}$.

Organizing equation to eliminate $\beta_{i}$, we can get

$$
\left\{\begin{array}{l}
\rho_{i 1}=\frac{-b_{1}+\sqrt{b_{1}^{2}-4 a_{1} c_{1}}}{2 a_{1}}, \\
\rho_{i 2}=\frac{-b_{1}-\sqrt{b_{1}^{2}-4 a_{1} c_{1}}}{2 a_{1}},
\end{array}\right.
$$

where

$a_{1}=1, b_{1}=-2 M \cos \alpha_{i}-2 N \sin \alpha_{i}, c_{1}=M^{2}+N^{2}-L_{2}^{2}, M=$ $X_{p}-X_{A_{i}}-L_{3} \cos \gamma_{i} N=Y_{p}-Y_{A_{i}}-L_{3} \sin \gamma_{i}$ 


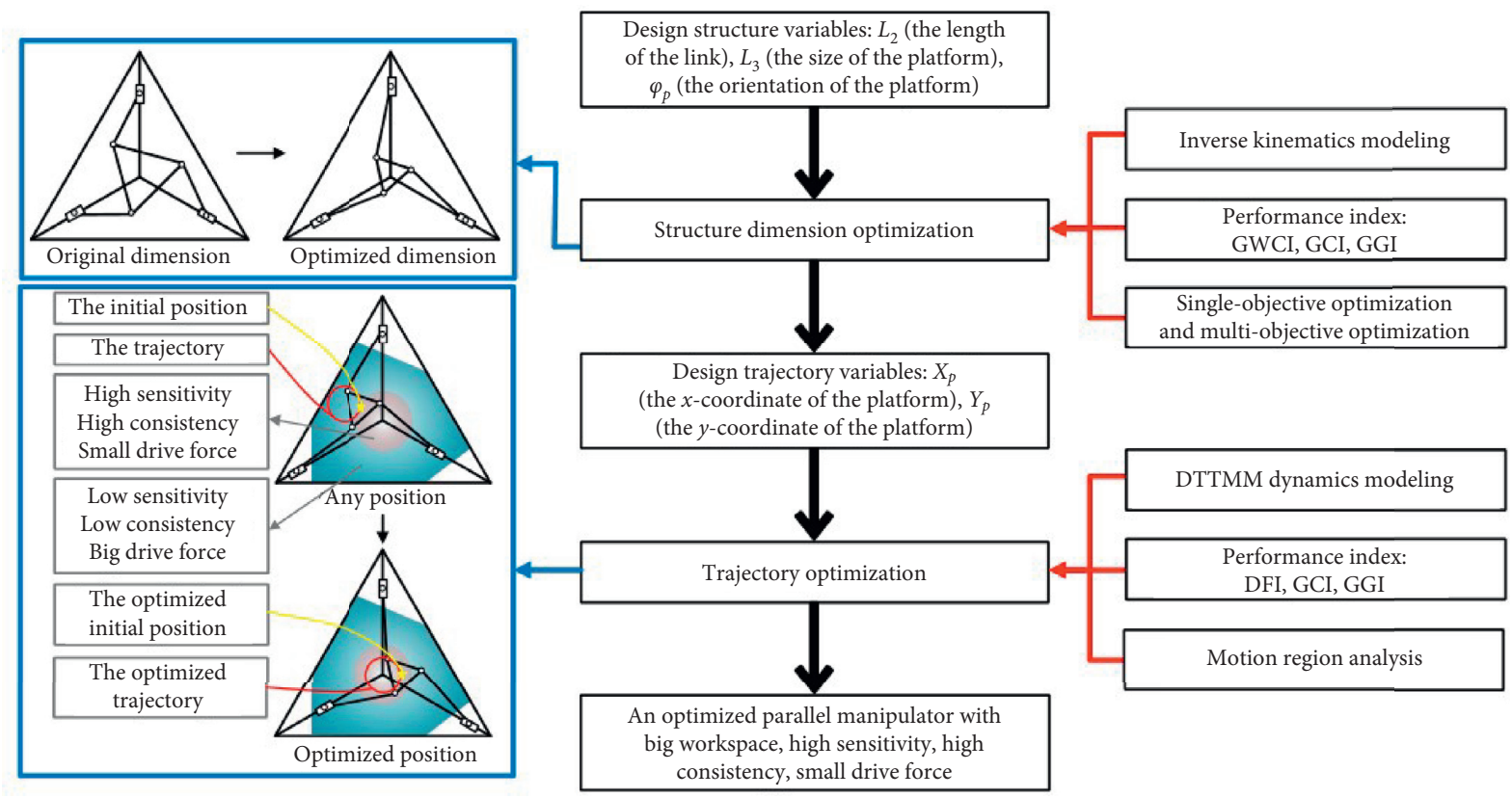

FIgURE 1: Schematic diagram for the design and optimization with integrated dynamics of a parallel robot manipulator.

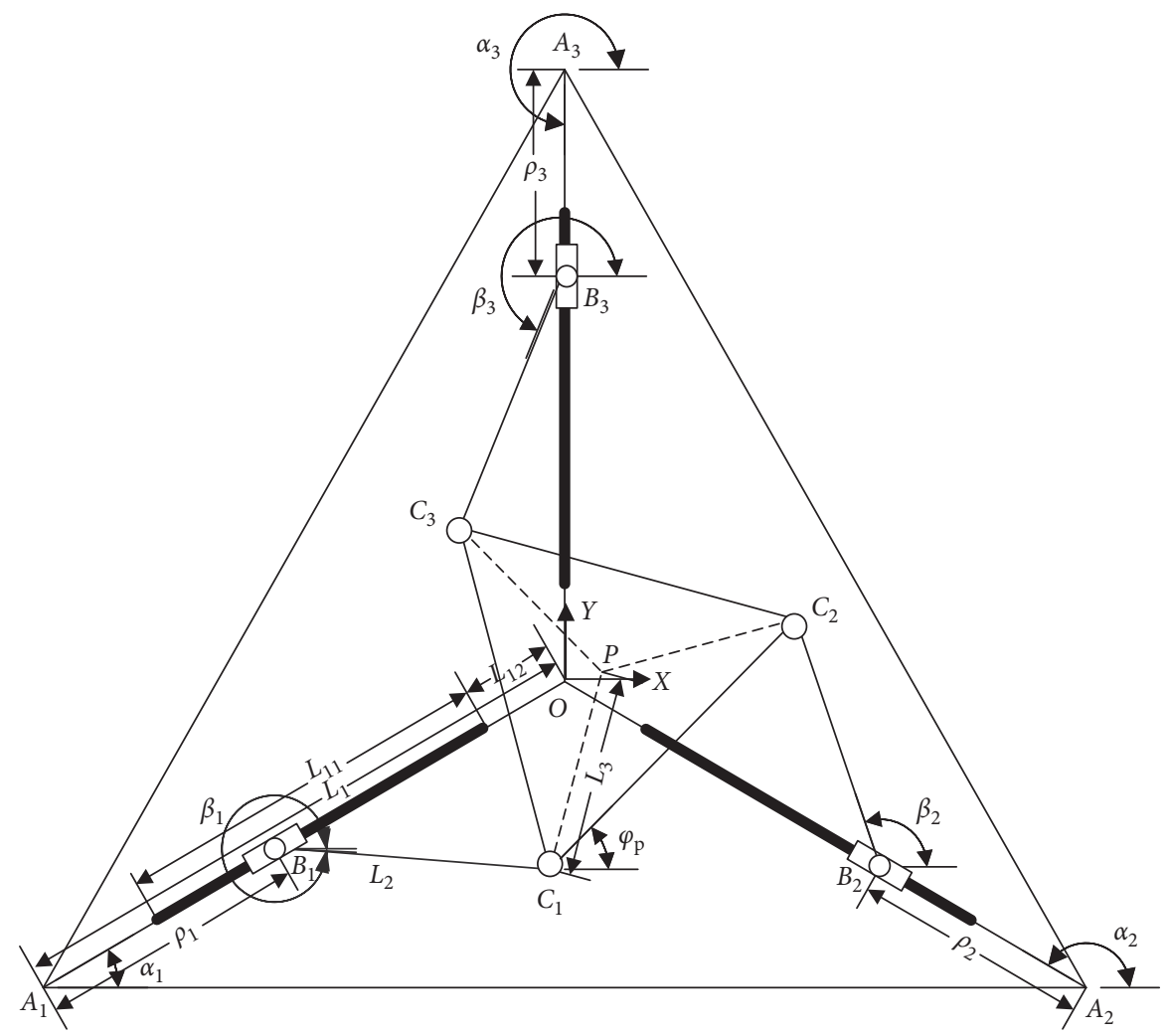

Figure 2: Configuration and coordinate system of the planar manipulator. 


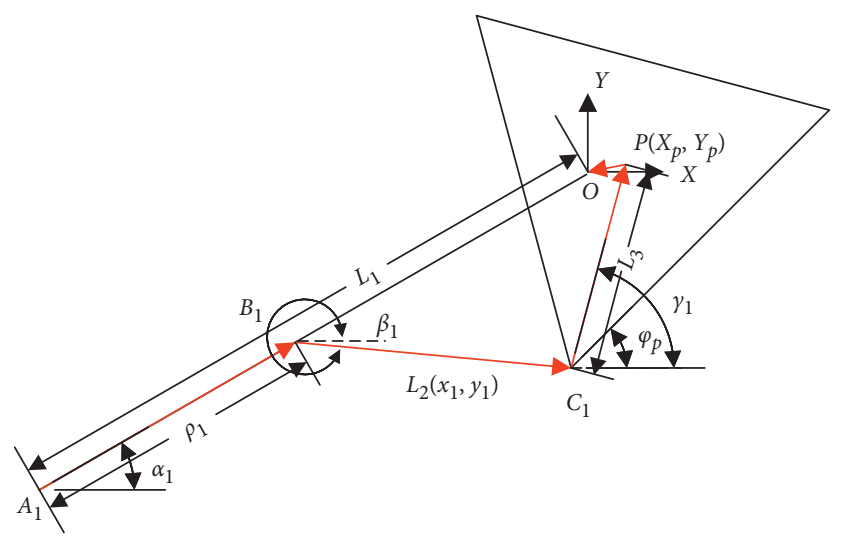

Figure 3: Schematic diagram of the close loop equation.

Organizing equation to eliminate $\rho_{i}$, we can get

$$
\left\{\begin{array}{l}
\beta_{i 1}=2 \arctan \frac{-b_{2}+\sqrt{b_{2}^{2}-4 a_{2} c_{2}}}{2 a_{2}}, \\
\beta_{i 2}=2 \arctan \frac{-b_{2}-\sqrt{b_{2}^{2}-4 a_{2} c_{2}}}{2 a_{2}},
\end{array}\right.
$$

where $a_{2}=M \sin \alpha_{i}-N \cos \alpha_{i}+L_{2} \sin \alpha_{i}, b_{2}=2 L_{2} \cos \alpha_{i}$, $c_{2}=M \quad \sin \alpha_{i}-N \cos \alpha_{i}-L_{2} \sin \alpha_{i}, M=X_{p}-X_{A_{i}}-L_{3}$ $\cos \gamma_{i,} N=Y_{p}-Y_{A_{i}}-L_{3} \sin \gamma_{i}$

Taking the derivative of equation (2), and getting rid of the $\dot{\beta}_{i}$, we have

$$
\begin{aligned}
& \dot{X}_{P} \cos \beta_{i}+\dot{Y}_{P} \sin \beta_{i}+\dot{\varphi}_{p} L_{3}\left[\sin \gamma_{i} \cos \beta_{i}-\cos \gamma_{i} \sin \beta_{i}\right] \\
& \quad=\dot{\rho}_{i}\left(\cos \alpha_{i} \cos \beta_{i}+\sin \alpha_{i} \sin \beta_{i}\right) .
\end{aligned}
$$

Simplifying equation (5), it can be written as $A \dot{X}=B \dot{\rho}$,

$$
A=L_{2}\left[\begin{array}{ccc}
\cos \beta_{1} & \sin \beta_{1} & L_{3} \sin \left(\frac{\pi}{6}+\varphi_{p}-\beta_{1}\right) \\
\cos \beta_{2} & \sin \beta_{2} & L_{3} \sin \left(\frac{5 \pi}{6}+\varphi_{p}-\beta_{2}\right) \\
\cos \beta_{3} & \sin \beta_{3} & L_{3} \sin \left(\frac{3 \pi}{2}+\varphi_{p}-\beta_{3}\right)
\end{array}\right],
$$

$$
B=L_{2}\left[\begin{array}{ccc}
\cos \left(\beta_{1}-\frac{\pi}{6}\right) & 0 & 0 \\
0 & \cos \left(\beta_{2}-\frac{5 \pi}{6}\right) & 0 \\
0 & 0 & \cos \left(\beta_{3}-\frac{3 \pi}{2}\right)
\end{array}\right] \text {, }
$$

$\dot{X}=\left(\begin{array}{lll}\dot{X}_{p} & \dot{Y}_{p} & \dot{\varphi}_{p}\end{array}\right)^{T}$,

$\dot{\rho}=\left(\begin{array}{lll}\dot{\rho}_{1} & \dot{\rho}_{2} & \dot{\rho}_{3}\end{array}\right)^{T}$.

In equation (6), $A$ is the output Jacobian matrix, $B$ is the input Jacobian matrix, which can be written in a uniform form as

$$
\dot{X}=J \dot{\rho},
$$

where $J=A^{-1} B . J$ is the velocity Jacobian transfer matrix of a 3-PRR planar parallel manipulator $[32,33]$.

\section{Dynamics Modeling with DT-TMM}

In the current literature, the dynamics equations of robot manipulators were usually established with traditional dynamic modeling methods, such as the Lagrange equation, Newton-Euler equation, Kane method, and the virtual work principle. However, the size of the global dynamic equations increases with the degrees of freedom and the number of divided subparts of each component. In this paper, DT-TMM dynamics is employed to establish the dynamic equations of the 3-PRR PPM to increase computation efficiency. To verify the applicability and effectiveness of the proposed DT-TMM for the dynamic modeling of parallel manipulators, numerical simulations are performed with the DT-TMM, the virtual work principle, and the ADMAS modeling methods, respectively, and numerical results are compared with each other.

3.1. Dynamic Modeling with Virtual Work Principle. Implementing the virtual work principle on the 3-PRR parallel planar manipulator, we have

$$
\delta \vec{\rho}^{T} F_{a}+\delta \vec{X}_{p}^{T} F_{p}+\delta \vec{\rho}^{T} F_{s}+\sum_{i=1}^{3} \delta \vec{X}_{i}^{T} F_{i}=0,
$$

where $\delta \vec{X}_{p}, \delta \vec{\rho}=J_{p} \delta \vec{X}_{p}$, and $\delta \vec{X}_{i}=J_{\beta_{i}} \delta \vec{X}_{p}$ are the virtual displacement of $\vec{X}_{p}, \vec{\rho}, \vec{X}_{i}$, respectively. $F_{a}=$ $\left(F_{a 1}, F_{a 2}, F_{a 3}\right)$ represents the forces applied on the slider, and $F_{p}=\left(-m_{p} \ddot{x}_{p},-m_{p} \ddot{y}_{p},-I_{p} \ddot{\varphi}_{p}\right)^{T}$ represents the inertia force of the end effector, in which $m_{p}$ and $I_{p}$ are the mass and the moment of inertia of the platform, respectively. $F_{s}=$ $\left(-m_{s 1} \ddot{\rho}_{1},-m_{s 2} \ddot{\rho}_{2},-m_{s 3} \ddot{\rho}_{3}\right)^{T}$ is the inertia force of the sliders, where $m_{s i}$ is the mass of the ith slider, $F_{i}=\left(-m_{i} \ddot{x}_{i}\right.$, $\left.-m_{i} \ddot{y}_{i},-I_{i} \ddot{\beta}_{i}\right)^{T}$ is the inertia force of the ith link, and $m_{i}$ and $I_{i}$ are the mass and the moment of inertia of the ith link. Substituting the virtual displacement into equation (10), the dynamics equation can be expressed as

$$
F_{a}=-F_{s}-\left(J_{p}^{T}\right)^{-1}\left(F_{p}+\sum_{i=1}^{3} J_{\beta i}^{T} F_{i}\right) .
$$

3.2. DT-TMM. In this section, the DT-TMM is employed to establish the multibody dynamics model of the 3-PRR PPM. The dynamic modeling based on the DT-TMM includes the following major steps. (1) The PPM is divided into components/elements. (2) The dynamics of each component/ element is derived through the Newton-Euler equation, and the kinematics of each component/element is established based on the inverse kinematics of the PPM. (3) The kinematics and dynamics of each component/element are linearized to obtain its transfer matrix based on the defined 
state vector at the inboard and outboard. (4) The dynamic model of the PPM is formulated by assembling the dynamics of each component/element through connecting the component/element transfer matrix [34].

3.2.1. State Vector and Transfer Matrices. In the DT-TMM, the state vector needs to be defined. Since the 3-PRR PPM is a chain system with planar motion, the state vector of the connection point between any rigid body and hinge is defined as

$$
z=\left[x, y, \theta, M_{z}, q_{x}, q_{y}, 1\right]^{T},
$$

where $x$ and $y$ are the position coordinates of the connection point for the global inertial coordinate system, $\theta$ is the orientation angle of the body, $M_{z}, q_{x}, q_{y}$ are the corresponding internal torques and internal forces in the same reference system, respectively.

The transfer matrix of a single element can be obtained by linearizing the dynamic equation of the element with the numerical integration method:

$$
z_{i+1}\left(t_{k}\right)=U_{i}\left(t_{k}\right) z_{i}\left(t_{k}\right) .
$$

In equation (13), the matrix $U_{i}\left(t_{k}\right)(i=1,2, \ldots, n)$ is the transfer matrix of the ith element which expresses the relationship between the state vectors of its output end $z_{i+1}$ and input end $z_{i}$ at time instant $t_{k}$. The size of the transfer matrices is always $(7 \times 7)$ for any multi-rigid-body systems moving in the plane.

Connecting all the components of the system, component by component, the whole system can be assembled and calculated as

$$
\begin{aligned}
z_{n} & =U_{\text {sys }} z_{1}, \\
U_{\text {sys }} & =U_{n}, U_{n-1} \cdots U_{2} U_{1},
\end{aligned}
$$

where the subscript " $n$ " denotes the total number of the elements of a chain multi-rigid-flexible-body system.

3.2.2. Transfer Matrices of Typical Elements. The 3-PRR PPM can be decomposed into three sliders, three intermediate links, one moving platform, and six revolute joints (Figure 4). With the inverse kinematics of the manipulator and the Newton-Euler equation, we can derive the transfer matrix for each decomposed component of the 3-PRR PPM.

If the size of the rigid body slider is ignored (Figure 5), the slider element can be treated as a point mass $m_{s}$. We have $x_{O}=x_{I}, y_{O}=y_{I}, \theta_{O}=\theta_{I}, M_{O}=M_{I}$.

Based on the Newton-Euler equation, the force equations can be given as

$$
q_{x, I}-q_{x, O}=-m_{s} \ddot{\rho}_{x}, q_{y, I}-q_{y, O}=-m_{s} \ddot{\rho}_{y} .
$$

To linearize all the terms, the transfer matrix of the slider is expressed as

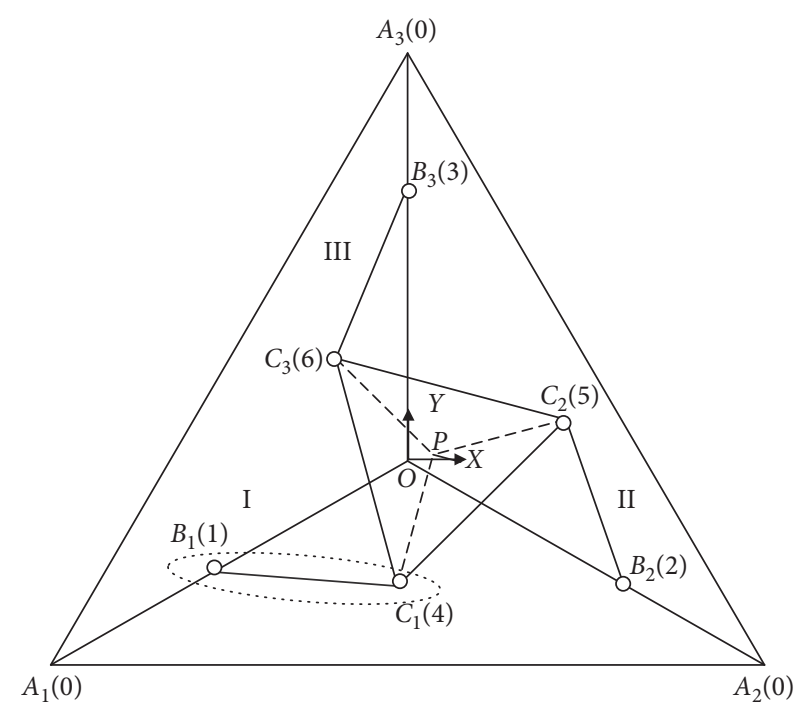

FIGURE 4: 3-PRR DT-TMM model.

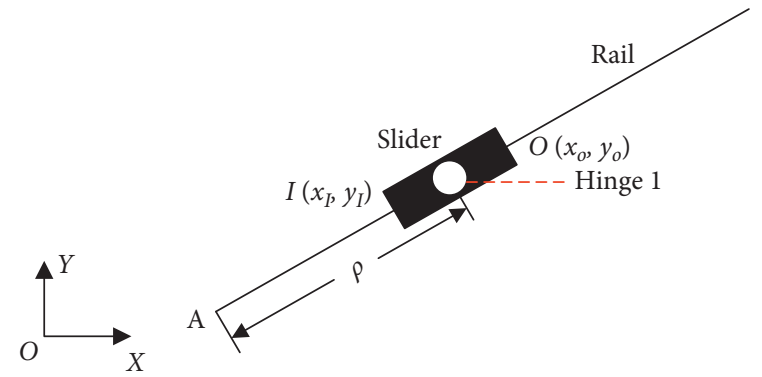

FIgURE 5: The slider model.

$$
U_{\text {slider }}=\left[\begin{array}{ccccccc}
1 & 0 & 0 & 0 & 0 & 0 & 0 \\
0 & 1 & 0 & 0 & 0 & 0 & 0 \\
0 & 0 & 1 & 0 & 0 & 0 & 0 \\
0 & 0 & 0 & 1 & 0 & 0 & 0 \\
0 & 0 & 0 & 0 & 1 & 0 & u_{57} \\
0 & 0 & 0 & 0 & 0 & 1 & u_{67} \\
0 & 0 & 0 & 0 & 0 & 0 & 1
\end{array}\right] \text {, }
$$

where $u_{57}=m_{s} \ddot{\rho}_{x}, u_{67}=m_{s} \ddot{\rho}_{y}$.

Each intermediate linkage used in this paper is assumed to be a rigid body (Figure 6), and the kinematic relation between its input and output is given as $x_{O}=x_{I}+l \cos \beta$, $y_{O}=y_{I}+l \sin \beta, \theta_{O}=\theta_{I}$. The moment balance regarding the inboard point yields

$$
\begin{aligned}
M_{O}= & M_{I}-l \sin \beta q_{x, I}+l \cos \beta q_{y, I}+\frac{1}{2} m_{l} l \cos \beta \ddot{y}_{I} \\
& -\frac{1}{2} m_{l} l \sin \beta \ddot{x}_{I}+J_{i} \ddot{\beta}+m_{l} \ddot{x}_{c} l \sin \beta-m_{l} \ddot{y}_{c} l \cos \beta,
\end{aligned}
$$




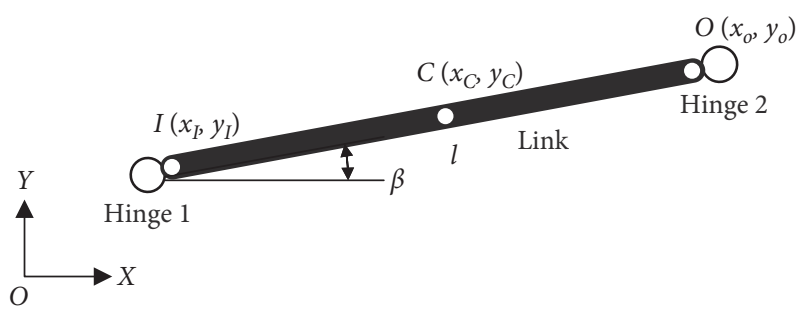

FIGURE 6: The intermediate linkage model.

where $\beta$ is the rotation angle of the linkage, $m_{l}$ is the mass of the linkage, $l$ is the length of the linkage, $\ddot{x}_{I}$ and $\ddot{y}_{I}$ are the input acceleration components, $\ddot{x}_{c}$ and $\ddot{y}_{c}$ are the acceleration components of the linkage mass center, $J_{i}$ is the inertia moment of the linkage.

The force equations of an intermediate linkage can be given based on the Newton-Euler equation:

$$
\begin{aligned}
& q_{x, I}-q_{x, O}=m_{l} \ddot{x}_{c}, \\
& q_{y, I}-q_{y, O}=m_{l} \ddot{y}_{c} .
\end{aligned}
$$

Linearizing the kinematic and the dynamic equations (the moment and force equations), the transfer matrix of each intermediate link is expressed as

$$
U_{\text {link }}=\left[\begin{array}{ccccccc}
1 & 0 & 0 & 0 & 0 & 0 & u_{17} \\
0 & 1 & 0 & 0 & 0 & 0 & u_{27} \\
0 & 0 & 1 & 0 & 0 & 0 & 0 \\
0 & 0 & 0 & 1 & u_{45} & u_{46} & u_{47} \\
0 & 0 & 0 & 0 & 1 & 0 & u_{57} \\
0 & 0 & 0 & 0 & 0 & 1 & u_{67} \\
0 & 0 & 0 & 0 & 0 & 0 & 1
\end{array}\right] \text {, }
$$

where $\quad u_{17}=l \cos \beta, \quad u_{27}=l \sin \beta, \quad u_{45}=-u_{27}, \quad u_{46}=u_{17}$, $u_{47}=(1 / 2) m_{l} l \cos \beta \ddot{y}_{I}-(1 / 2) m_{l} l \sin \beta \ddot{x}_{I}+J_{i} \ddot{\beta}+m_{l} \ddot{x}_{c} l \sin \beta-$ $m_{l} \ddot{y}_{c} l \cos \beta, u_{57}=-m_{l} \ddot{x}_{c}, u_{67}=-m_{l} \ddot{y}_{c}$.

A hinge connects two objects (Figure 7), and it allows to rotate freely and is a passive joint. Its input and output parameters are the same, then the transfer matrix is:

$$
U_{\text {hinge }}=\left[\begin{array}{lllllll}
1 & 0 & 0 & 0 & 0 & 0 & 0 \\
0 & 1 & 0 & 0 & 0 & 0 & 0 \\
0 & 0 & 1 & 0 & 0 & 0 & 0 \\
0 & 0 & 0 & 1 & 0 & 0 & 0 \\
0 & 0 & 0 & 0 & 1 & 0 & 0 \\
0 & 0 & 0 & 0 & 0 & 1 & 0 \\
0 & 0 & 0 & 0 & 0 & 0 & 1
\end{array}\right] .
$$

3.2.3. Transfer Matrices of 3-PRR PPM. The 3-PRR PPM can be treated as a branched system in which each kinematic chain is a subchain system and the moving platform is a rigid body with three inboards at tips 4, 5, 6, and one outboard at its mass center $P$ (Figure 8 ). The transfer matrix of the ith kinematic chain is given as

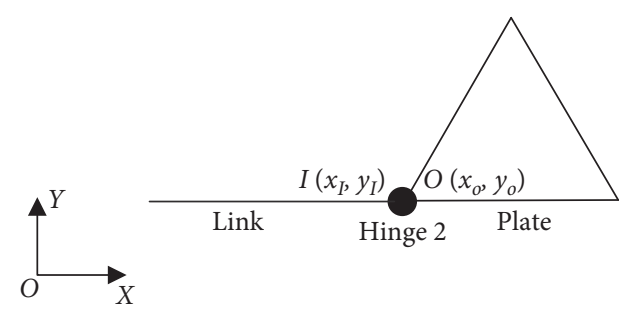

Figure 7: The hinge model.

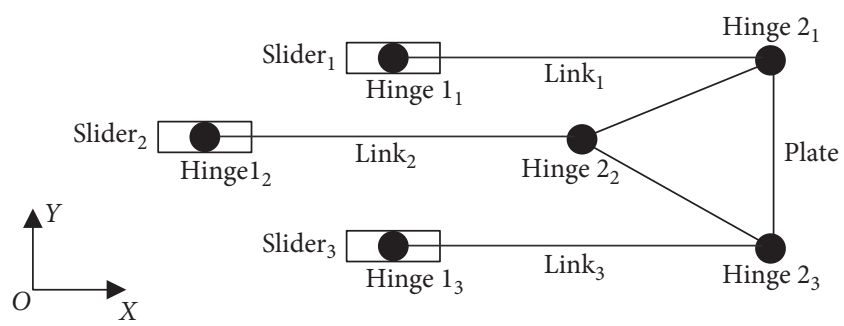

FIgURE 8: Transfer Matrix with branch system model for the 3-PRR planar parallel manipulator.

$$
U_{i}=U_{\text {hingel }_{i}} U_{\text {link }_{i}} U_{\text {hinge }_{i}} U_{\text {slider }_{i}}, \quad i=1,2,3,
$$

where $U_{\text {slider }_{i}}$ is the transfer matrix of the slider which is point mass, $U_{\text {hingel }_{i}}$ and $U_{\text {hinge }}$ are the smooth pin hinges connected at both tips of the rigid linkages and $U_{\text {link }_{i}}$ is the rigid beam transfer matrix represents the $i$ th linkage. The state vectors for each chain system is expressed as

$$
\begin{aligned}
& \vec{z}_{1,2,3}=\left[\begin{array}{lllllll}
x & y & 0 & 0 & q_{x} & q_{y} & 1
\end{array}\right]_{1,2,3} \\
& \vec{z}_{4,5,6}=\left[\begin{array}{lllllll}
x & y & 0 & 0 & q_{x} & q_{y} & 1
\end{array}\right]_{4,5,6}
\end{aligned}
$$

So the overall transfer equation for each chain system is

$$
\vec{z}_{i+3}=U_{i} \vec{z}_{i}, \quad i=1,2,3 \text {. }
$$

The dynamic equations of the platform can be written based on the Newton-Euler equation as

$$
\begin{aligned}
& \sum F_{x}=\ddot{x}_{p} m_{p}, \\
& \sum F_{y}=\ddot{y}_{p} m_{p}, \\
& \sum M=\ddot{\varphi}_{p} I_{p} .
\end{aligned}
$$

Using the kinematic equations, equation (24) can be combined and rewritten in a matrix form as

$$
\left[\begin{array}{rrrrrr}
1 & 0 & 1 & 0 & 1 & 0 \\
0 & 1 & 0 & 1 & 0 & 11 \\
-d_{y 1} & d_{x 1} & -d_{y 2} & d_{x 2} & -d_{y 3} & d_{x 3}
\end{array}\right]\left[\begin{array}{c}
q_{x 4} \\
q_{y 4} \\
q_{x 5} \\
q_{y 5} \\
q_{x 6} \\
q_{y 6}
\end{array}\right]=\left[\begin{array}{c}
\ddot{x}_{p} m_{p} \\
\ddot{y}_{p} m_{p} \\
\varphi_{p} I_{p}
\end{array}\right],
$$


where $\quad d_{x i}=-(\sqrt{3} / 3) l_{p} \cos \gamma_{i}, \quad d_{y i}=(\sqrt{3} / 3) l_{p} \sin \gamma_{i}$, $\left[\begin{array}{lll}\gamma_{1} & \gamma_{2} & \gamma_{3}\end{array}\right]=\left[\begin{array}{lll}210 & -30 & 90\end{array}\right]$, and $q_{x i}, q_{y i}, i=1,2,3$ are internal forces at outboard tips of linkages.

Equation (25) gives three equations to solve six unknowns. To solve the dynamic equations, the relations of internal forces $q_{x i}$ and $q_{y i}$ are required to be established. From the transfer equation of each subsystem (equation (23)), we have

$$
\left[\begin{array}{c}
x \\
y \\
0 \\
0 \\
q_{x} \\
q_{y} \\
1
\end{array}\right]_{i+3}=\left[\begin{array}{lllllll}
u_{11} & u_{12} & u_{13} & u_{14} & u_{15} & u_{16} & u_{17} \\
u_{21} & u_{22} & u_{23} & u_{24} & u_{25} & u_{26} & u_{27} \\
u_{31} & u_{32} & u_{33} & u_{34} & u_{35} & u_{36} & u_{37} \\
u_{41} & u_{42} & u_{43} & u_{44} & u_{45} & u_{46} & u_{47} \\
u_{51} & u_{52} & u_{53} & u_{54} & u_{55} & u_{56} & u_{57} \\
u_{61} & u_{62} & u_{63} & u_{64} & u_{65} & u_{66} & u_{67} \\
0 & 0 & 0 & 0 & 0 & 0 & 1
\end{array}\right]_{i}\left[\begin{array}{c}
x \\
y \\
0 \\
0 \\
q_{x} \\
q_{y} \\
1
\end{array}\right]_{i}
$$

The fourth row of the transfer matrix provides the moment balance relations so that the internal forces along the $y$-direction at 1,2,3 can be written as the functions of the internal forces along $x$-direction as

$$
q_{y i}=-\frac{1}{u_{46, i}}\left[\begin{array}{lll}
u_{41} & u_{42} & u_{43}
\end{array}\right]_{i}\left[\begin{array}{l}
x \\
y \\
1
\end{array}\right]_{i}-\frac{u_{45, i}}{u_{46, i}} q_{x i}, \quad i=1,2,3 .
$$

Inserting equation (27) into the fifth and sixth rows in equation (26), $q_{x}$ and $q_{y}$ at point $i+3$ where $i=1,2,3$ can be expressed only as the functions of inboard internal forces $q_{x i}$ as

$$
\left[\begin{array}{l}
q_{x} \\
q_{y}
\end{array}\right]_{i+3}=\left[\begin{array}{lllllll}
u_{51} & u_{52} & u_{53} & u_{54} & u_{55} & u_{56} & u_{57} \\
u_{61} & u_{62} & u_{63} & u_{64} & u_{65} & u_{66} & u_{67}
\end{array}\right]_{i}\left[\begin{array}{c}
q_{x} \\
q_{y}\left(q_{x}\right)
\end{array}\right]_{i},
$$

where $q_{y}\left(q_{x}\right)$ represents that the $q_{y}$ is a function of $q_{x}$ at the inboard $i$. Substituting equation (28) into equation (25), we can get

$$
\left[\begin{array}{cccccc}
1 & 0 & 1 & 0 & 1 & 0 \\
0 & 1 & 0 & 1 & 0 & 1 \\
-d_{y 1} & d_{x 1} & -d_{y 2} & d_{x 2} & -d_{y 3} & d_{x 3}
\end{array}\right]\left[\begin{array}{c}
q_{y 4}\left(q_{x 1}\right) \\
q_{y 4}\left(q_{x 1}\right) \\
q_{x 5}\left(q_{x 2}\right) \\
q_{y 5}\left(q_{x 2}\right) \\
q_{x 6}\left(q_{x 3}\right) \\
q_{y 6}\left(q_{x 3}\right)
\end{array}\right]=\left[\begin{array}{c}
\ddot{x}_{p} m_{p} \\
\ddot{y}_{p} m_{p} \\
\ddot{\varphi}_{p} I_{p}
\end{array}\right] .
$$

Equation (25) has been changed to equation (29) that only contains three unknowns. The actuated forces on $x$-direction applied on sliders can be obtained from equation (29), and the forces along the $y$-direction can be calculated from equation (27).

3.3. Dynamic Simulation and Verification. To verify the dynamic modeling using the DT-TMM, numerical simulations are conducted on the 3-PRR PPM and compared with the results of the dynamic modeling using the virtual work principle and ADAMS. In all simulations, the orientation angle of the moving platform remains unchanged, with the setting $\varphi_{p}=45^{\circ}$. The center position of the moving platform moves periodically along the circle with radius $2 \mathrm{~cm}$, and the moving platform trajectory is defined as

$$
\begin{array}{ll}
X_{p}=P_{x 0}+2 \cos (\pi t), & 0 \leq t \leq 4 \mathrm{~s}, \\
Y_{p}=P_{y 0}+2 \sin (\pi t), & 0 \leq t \leq 4 \mathrm{~s},
\end{array}
$$

where $P_{x 0}=0$ and $P_{y 0}=0$ are the initial position coordinate at the mass center of the end effector. The trajectory is set in a safe area such that the manipulator works without any singularity in two periods $(4 \mathrm{~s})$. The dynamics parameters for the 3-PRR PPM are listed in Table 1.

In the DT-TMM method, since the trajectory at the mass center of the platform as well as the kinematic equations of all three chains are given in the previous instant. The positions and accelerations of platform $x_{p}, y_{p}, \ddot{x}_{p}, \ddot{y}_{p}, \ddot{\varphi}_{p}$ and positions of sliders are known. From equation (29), the actuated forces could be solved. During the ADAMS simulation (Figure 9), the kinematics parameters of the slider are obtained by giving the trajectory of the center of the moving platform. Taking the spline curve of slider displacement as the input of the moving drive, the driving force required by a parallel manipulator can be obtained (Figure 10). The simulation has been carried out with time interval $\Delta T=0.01 \mathrm{~s}$. The root mean square error (RMSE) is used to evaluate the driving forces of different methods. Compared with the two methods of DT-TMM and ADAMS simulation, the RMSE value for all three driving forces is only 0.0018 , the calculated order of magnitude is smaller, which means the two methods are basically identical. The RMSE of the DT-TMM model and the virtual work principle model is 0 , which means that the results are completely consistent (Table 2). The numerical simulations and comparison verify the effectiveness of the DT-TMM model for the dynamic modeling of the 3-PRR PPM. The driving force of the manipulator increases with the increase of the speed and acceleration of the end platform. In the case of the same path, it is necessary to provide more driving force to realize the high-speed and high-acceleration characteristics of the manipulator. The size of the driving force depends on the motor and the application. For example, the rated torque of the motor selected for the test bench is $0.2 \mathrm{Nm}$ and the screw pitch of the lead screw is $2 \mathrm{~mm}$. Then, the driving force of the motor is around $628.3 \mathrm{~N}$ with the consideration of the gearbox. As shown in Figure 11, in order to achieve a circle with the same radius of $2 \mathrm{~cm}$, the smaller the rotation cycle of the platform, the greater the motor driving force required. This means that testing a faster trajectory requires larger driving force. Therefore, with the selected testing motors, the shortest rotation cycle of the moving platform is 0.01 second as indicated in Figure 11.

One main contribution of this part is to extend the DTTMM into the dynamic modeling of PPMs and to compare it with the traditional calculation methods and the ADAMS modeling. The DT-TMM method avoids the large size of 
TABLE 1: Parameters of the 3-PRR PPM.

\begin{tabular}{llc}
\hline Symbols & Unit & Parameters \\
\hline$L_{1}=10 \sqrt{3}$ & $(\mathrm{~cm})$ & The distance from the origin to $A$ \\
$L_{2}=8$ & $(\mathrm{~cm})$ & Length of the intermediate link \\
$L_{3}=(10 \sqrt{3} / 3)$ & $(\mathrm{cm})$ & The distance from the origin to $C$ \\
$\varphi_{p}=45$ & $(\mathrm{deg})$ & The orientation of the platform \\
\hline
\end{tabular}

global dynamic equations by decomposing the global dynamics to the component dynamics and transferring the component dynamics from one component to its neighboring components. With the DT-TMM, the matrix size of the multibody system dynamic equations does not increase with the degrees of freedom of the multibody systems and hence significantly reduces the computation cost. This method provides a novel method for the dynamics study of PPMs and facilitates the following structure parameter and motion optimization of the PPM, dynamic analysis, and control of parallel robotic manipulators etc. because of its high computation efficiency.

\section{Structure Parameter Optimization}

The kinematic performance of a parallel robot manipulator heavily depends on its structure geometry parameters. Therefore, it is desirable to optimize the structure geometry parameters based on selected performance indices for each application. Various kinematic performance indices were proposed for the optimization of structural parameters for parallel manipulators in the literature. In this section, we briefly introduce how to define and analyze the workspace and singularity since both of them are the most important properties that are usually employed to define various kinematic performance indices. Then we select and define the kinematic performance indices of the presented PPM. Finally, we conduct the structure parameter optimization with single and multiple objectives.

4.1. Workspace. According to whether the orientation angle $\varphi_{p}$ changes or not, the workspace of planar parallel manipulators is divided into the reachable workspace and constant orientation workspace [35]. Reachable workspace is the set of all workspaces that can be reached by the mechanism when the feasible orientation angle is taken. Constant orientation workspace refers to the workspace accessible to the center position of the moving platform when the orientation $\varphi_{p}$ is a constant value, which is a subset of reachable workspace. In order to reduce the angle error caused by the rotation of the moving platform, the constant orientation workspace is considered in the design.

In the process of solving the workspace, the analytic method and the numerical method are generally adopted. The analytical method is largely dependent on the research results of the positional solution of the mechanism. It mainly employs various geometric methods. For example the Envelop Surface method takes each branch chain as a single open chain, then finds its respective envelope surface, and gets the reachable working space by intersecting the envelope surface of each branch chain [36]. The solving characteristic of geometric methods is to avoid complex mathematical operations and draw the workspace area directly through the software, but it is not accurate enough in the actual analysis. Numerical methods are widely used in the search process, including the Jacobian matrix method, limit boundary search method, network method, region search method, etc. This paper uses the region search method to solve the workspace. The region search method uses the kinematic inverse solution equation to determine whether there are real numbers as the judgment conditions [37, 38].

If the quadratic function of equations (3) and (4) obtains a real solution, then the driving distance $\rho_{i}$ and the link angle $\beta_{i}$ obtain a real solution, and the positional region corresponding to the real solution of $\rho_{i}$ and $\beta_{i}$ is the reachable workspace of the 3-PRR parallel positioning platform. Therefore, the reachable workspace of the 3-PRR parallel positioning platform can be drawn based on the discriminant formula $\Delta_{1}=b_{1}^{2}-4 a_{1} c_{1} \geq 0$ and $\Delta_{2}=b_{2}^{2}-4 a_{2} c_{2} \geq 0$ $[39,40]$. The collection of workspaces is given as

$$
\left\{(X, Y) \mid \Delta_{1}=b_{1}^{2}-4 a_{1} c_{1} \geq 0 \text { and } \Delta_{2}=b_{2}^{2}-4 a_{2} c_{2} \geq 0\right\}
$$

where $(X, Y)$ is the coordinate of the workspace, $\Delta_{1}$ is the discriminant of equation (4) after eliminating $\beta_{i}$, and $\Delta_{2}$ is the discriminant of equation (3) after eliminating $\rho_{i}$.

4.2. Singular Type. At singular configurations, the performance of the robot mechanism will deteriorate or even become uncontrollable, which is usually manifested as the locked joint or the loss of the degree of freedom at the end of the mechanism platform [41]. Whether the mechanism is at a singular configuration can be determined by whether the velocity Jacobian matrix of the mechanism is a singular matrix. According to the inverse kinematics (equation (6)), the input and output velocity Jacobian matrix can be obtained $A \dot{X}=B \dot{\rho}$ or $\dot{X}=J \dot{\rho}$. The analysis of each singular classification of the $3-$ PRR PPM is presented as follows [42-44]:

(1) The first singularity: When matrix $B$ is singular, the mechanism suddenly loses one or more degrees of freedom. By calculating the determinant of equation (8), it can be obtained that the singular case of the mechanism is $\beta_{1}=-(\pi / 3)$ or $(2 \pi / 3), \beta_{2}=(\pi / 3)$ or $(4 \pi / 3), \beta_{3}=\pi$ or $2 \pi$. In other words, when at least one link is perpendicular to the linear guideway, the mechanism appears singular. If the three links are perpendicular to the linear guideway, the mechanism is at the critical point of topological transformation. Therefore, when at least one or at most two links are perpendicular to the linear guideway (Figure 12), the end of the mechanism reaches the boundary of the workspace, which belongs to boundary singularity.

(2) The second singularity: When matrix $A$ is singular, even if the drive joint is locked, the end of the mechanism still has degrees of freedom. By calculating the determinant of equation (7), there are three kinds of singular situations (Figure 13). 


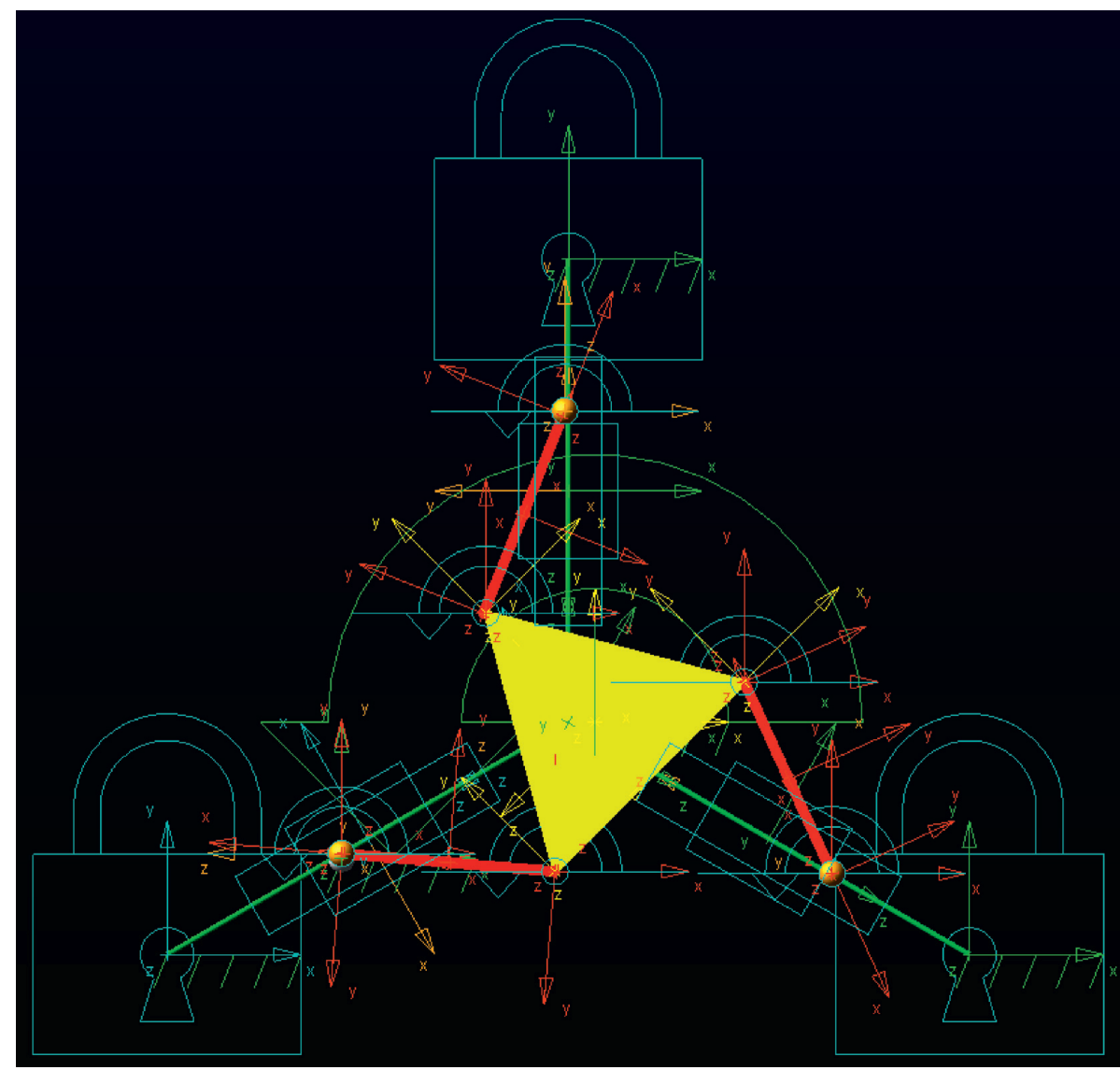

Figure 9: The ADAMS model of the manipulator.

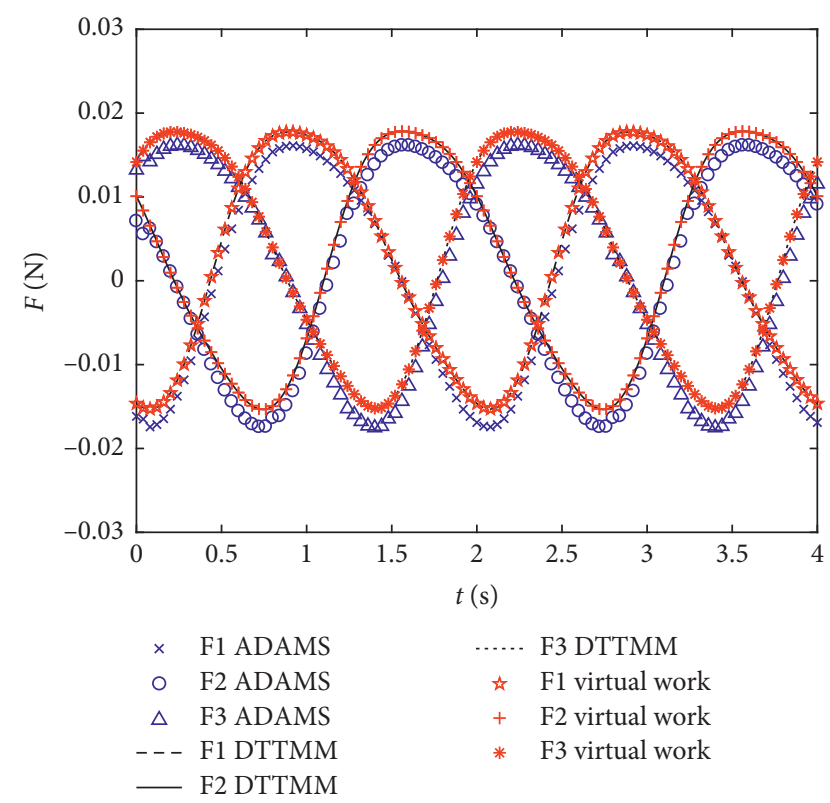

FIgURE 10: Compare the driving forces of the different methods.

Case 1. Two of the three links are collinear and the angle of the other link is $\beta_{i}=\varphi_{p}+(4 \pi i-3 \pi / 6) \mp k \pi$.

Case 2. All three links are parallel.
TABle 2: The root mean square error between the difference methods.

\begin{tabular}{lccc}
\hline Methods & F1 & F2 & F3 \\
\hline DT-TMM and ADAMS & 0.0018 & 0.0018 & 0.0018 \\
DT-TMM and virtual work & 0 & 0 & 0 \\
\hline
\end{tabular}

Case 3. The angles of the three links meet at the same time in the case of $\beta_{i}=\varphi_{p}+(4 \pi i-3 \pi / 6) \mp k \pi$, that is, the extension lines of the three links meet at one point.

(3) The third singularity: When the first and second kinds of singularities occur simultaneously in the mechanism, that is, when the matrix $J$ is singular, the distribution of the determinant of the Jacobian matrix $J$ can be plotted through the region search method, so as to obtain the distribution of the nonsingular workspace of the mechanism.

Through the analysis of the above three singular types, we can define the set of singular occurrence as

$$
\begin{aligned}
\left\{\beta_{i} \mid(\operatorname{det}(B)\right. & =0 \text { and } \operatorname{det}(A) \neq 0) \|(\operatorname{det}(A) \\
& =0 \text { and } \operatorname{det}(B) \neq 0) \|(\operatorname{det}(J)=0)\} .
\end{aligned}
$$




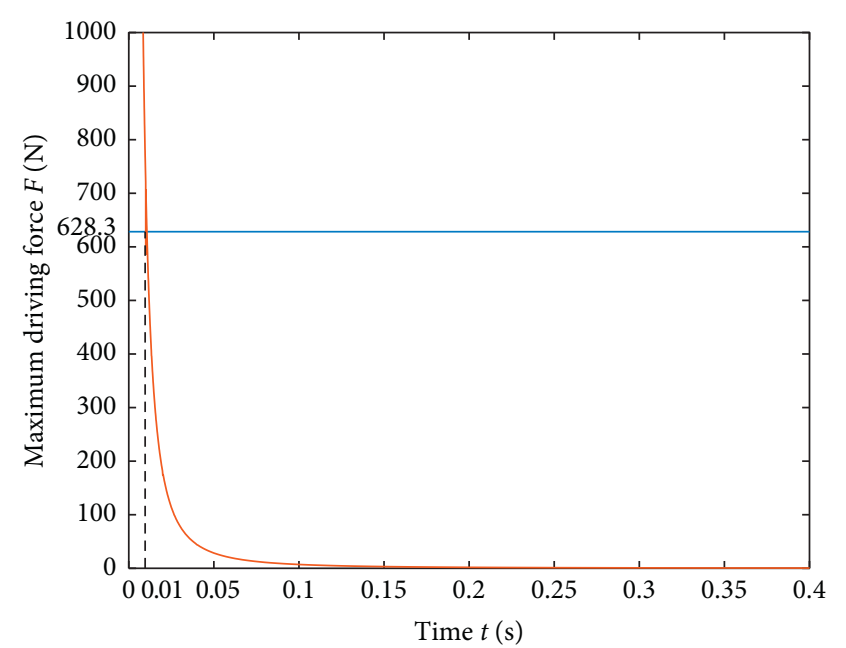

FIGURE 11: The relationship between the platform's motion period and the driving force.

4.3. Parameter Design. The 3-PRR planar parallel manipulator chooses a symmetrical configuration; that is, the center position of the moving platform is located at the origin of the coordinate system. Besides, the relevant parameters of the static platform of the planar parallel manipulator are fixed, as can be seen from Figure 2. The static side length is $30 \mathrm{~cm}$, the available guideway $L_{12}$ is $10 \mathrm{~cm}$, and the distance $L_{11}$ from the center point $O$ is $3 \mathrm{~cm}$. We assume that the given dimensions of the mechanical structure are the side length of the static platform, the position and the orientation angle of the linear guideway. The parameters to be optimized are the length of the link $L_{2}$, the size of the moving platform $L_{3}$, and the orientation $\varphi_{p}$ of the moving platform. Therefore, this paper mainly studies the influence of variables $L_{2}, L_{3}$, and $\varphi_{p}$ on the kinematic performance of the 3-PRR PPM mechanism, and the design variables are chosen as

$$
X=\left[L_{2}, L_{3}, \varphi_{p}\right] \text {. }
$$

Considering that the moving platform must be smaller than the static platform, and not too small to affect the rigidity of the moving platform, $L_{3}$ is limited to $[1.5 \mathrm{~cm}$, $6.5 \mathrm{~cm}$ ]. Since the position of the slide table is $3 \mathrm{~cm}$ away from the origin and the stroke of the slide track is $10 \mathrm{~cm}$, the size of $L_{2}$ is limited to $[3 \mathrm{~cm}, 13 \mathrm{~cm}]$. When the orientation $\varphi_{p}$ is 0 , the mechanism is in the third case of the second type of singularity (Figure 13), so the orientation $\varphi_{p}$ must be greater than $0^{\circ}$. To avoid the intersection between the link and the moving platform, the orientation within $90^{\circ}$ should be studied, so $\varphi_{p}$ is limited to $\left[5^{\circ}, 85^{\circ}\right]$. Besides, to meet the requirements of the above single parameters, the parallel manipulator must not be located at singular configurations for the optimization.

4.4. Performance Indices. The kinematic performance indexes of the robot include isotropy of the Jacobian matrix, speed and bearing capacity, stiffness, accuracy, workspace size, redundancy, and so on. In this paper, we are mainly concerned with integrating the kinematic into the

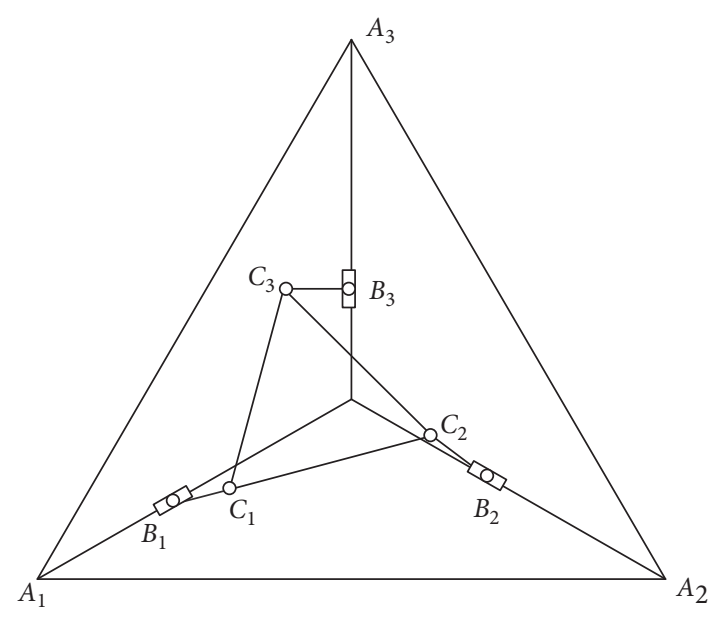

Figure 12: The first singularity (one link vertical guide).

optimization process by using the major kinematic performance indices: Global Workspace Conditioning Index (GWCI), Global Condition Index (GCI), and Global Gradient Index (GGI) as an illustration example to optimize the size parameters of the mechanism [45]. Through the single analysis and comprehensive analysis of these indicators, the optimal size parameters are achieved for the presented PPM.

4.4.1. Global Workspace Conditioning Index (GWCI). The size of the machine workspace represents the robot's range of activity, which is an important kinematic index to measure the robot's working ability. The workspace of the robot is defined as the set of all positions that can be reached by the end-effector under the limitation of structure. It is not possible to judge whether the work ability is good or bad only by the value of accessible workspace [46]. Therefore, the global workspace condition index (GWCI) is introduced

$$
\text { GWCI }=\frac{\iint_{S} \mathrm{~d} s}{\Delta x \Delta y}
$$

where $\Delta x, \Delta y$ are the difference between the upper and lower boundaries of $X$ and $Y$ of the selected region (SR) according to the requirements which must contain all workspaces. $\Delta x, \Delta y$ and SR are marked in Figure 14. $S$ is the workspace calculated by equation (31). The range of GWCI is 0 to 1 . The larger the GWCI value, the larger the workspace available and the stronger the working ability of the parallel planar robot. Therefore, GWCI must be maximized in the optimal design of the manipulator. Figure 14 shows the SR and Workspace for $L_{2}=8 \mathrm{~cm}, L_{3}=(10 \sqrt{3} / 3) \mathrm{cm}$, and $\varphi_{p}=45^{\circ}$, then $\mathrm{GWCI}=S / \mathrm{SR}=0.0918$ for the parameters. Figure 15 shows the relationship between design variables and GWCI.

4.4.2. Global Condition Index (GCI). Global Condition Index (GCI) is the reciprocal of condition number average throughout the workspace, and it can reflect the size of the institution of sensitivity in the working space of the average conditions [47]. To improve the sensitivity of planar parallel 


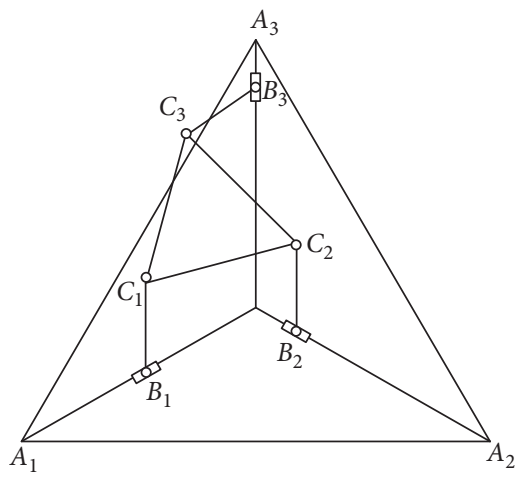

(a)

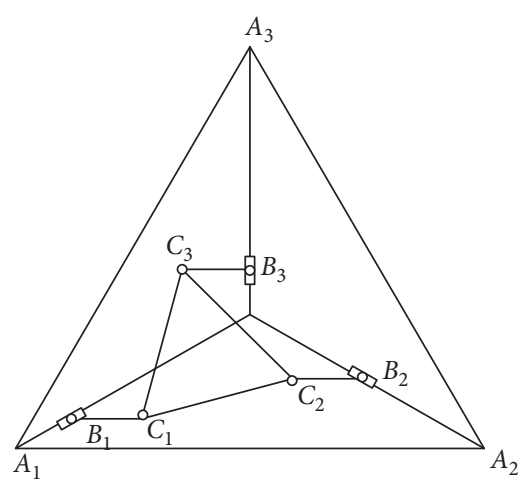

(b)

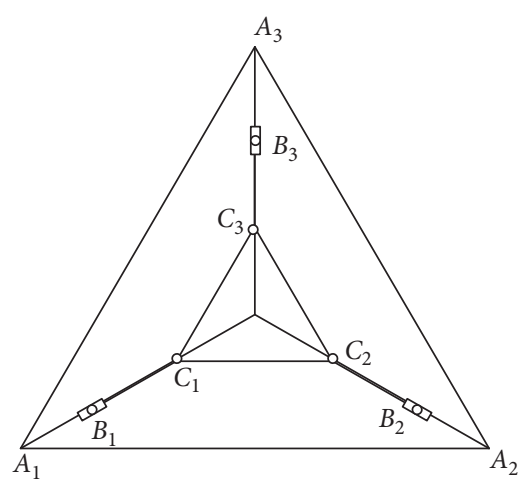

(c)

Figure 13: The second singularity. (a) Case 1. (b) Case 2. (c) Case 3.

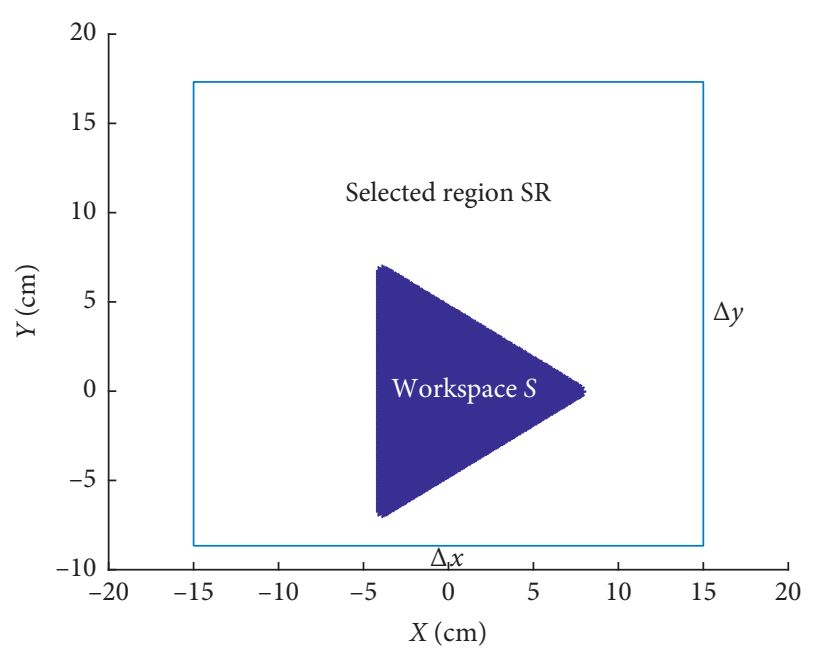

FIGURE 14: The selected region and Workspace for $L_{2}=8 \mathrm{~cm}, L_{3}=$ $(10 \sqrt{3} / 3) \mathrm{cm}$ and $\varphi_{p}=45^{\circ}$.

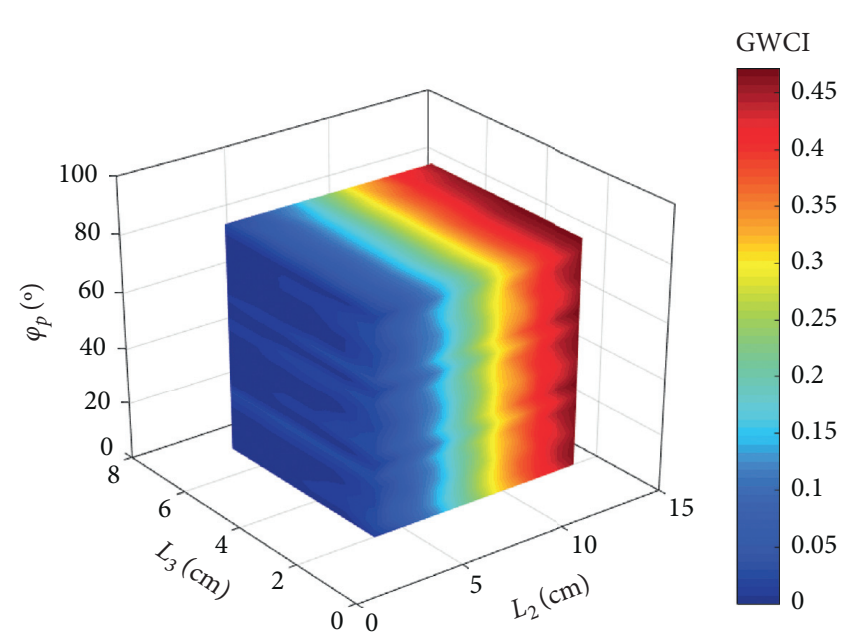

FIGURE 15: The relationship between the design variables and GWCI. robot moving platform, and make it have good isotropic in the workspace, GCI is chosen as the optimization index as [48]

$$
\mathrm{GCI}=\frac{\iint_{S}(1 / k(J)) \mathrm{d} s}{\iint_{S} \mathrm{~d} s,}
$$

where $k(J)$ is the local condition number of the Jacobian matrix. In the design of the robot mechanism, the Jacobian matrix of the mechanism should be as small as possible. In order to study the distribution of Jacobian condition number in the whole workspace in a controllable scope, we can take the reciprocal of condition number as an evaluation index, then the scope of GCI is 0 to 1 . The larger the GCI is, the better the maneuverability and sensitivity of the parallel robot become. Figure 16 shows the distribution of the reciprocal condition number $1 / k(J)$ of the Jacobian matrix for $L_{2}=8 \mathrm{~cm}, L_{3}=(10 \sqrt{3} / 3) \mathrm{cm}$, and $\varphi_{p}=45^{\circ}$, then $\mathrm{GCI}=$ 0.0610 is the integral of $1 / k(J)$ in the entire workspace divided by the workspace area. Figure 17 shows the relationship between the design variables and GCI.

4.4.3. Global Gradient Index (GGI). Global Gradient Index (GGI) is used to measure the uniformity of kinematics sensitivity. If the Jacobi condition number index of adjacent points drops sharply, these configuration parameters should not be taken [49]. To ensure consistency in the sensitivity of the workspace, the gradient value should be as small as possible.

$$
\mathrm{GGI}=\nabla(\mathrm{GCI})=\max _{S}\left\|\frac{\nabla 1}{k(J)}\right\|,
$$

where $S$ is the workspace and $k(J)$ is the local condition number of the Jacobian velocity matrix. The larger the GGI value is, the greater the fluctuation of kinematic sensitivity will be, which means that the kinematic performance of the parallel robot will fluctuate up and down in the whole workspace. When GGI is small, the kinematic performance of the mechanism in the workspace is stable. Therefore, a smaller global gradient exponent should be selected for the entire workspace. Figure 18 shows the relationship between the design variables and GGI. 


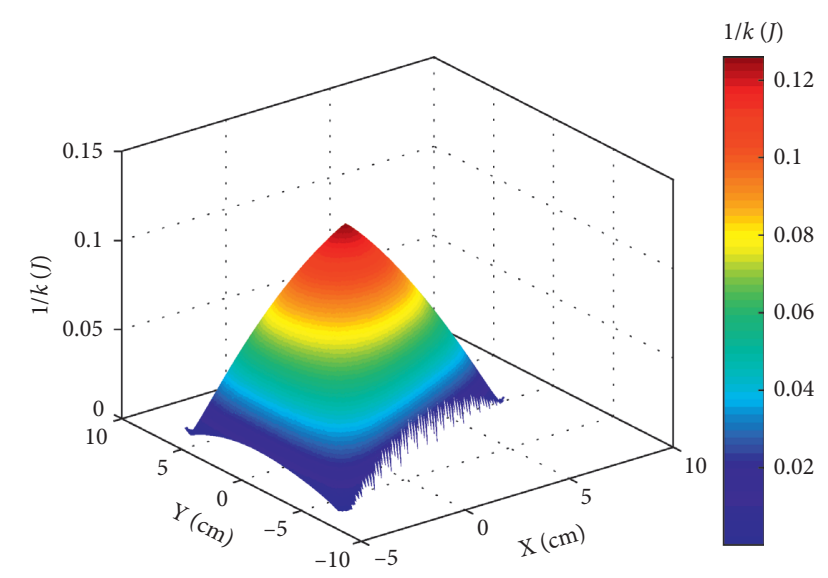

Figure 16: The distribution of $1 / k(J)$ for $L_{2}=8 \mathrm{~cm}$, $L_{3}=(10 \sqrt{3} / 3) \mathrm{cm}$, and $\varphi_{p}=45^{\circ}$.

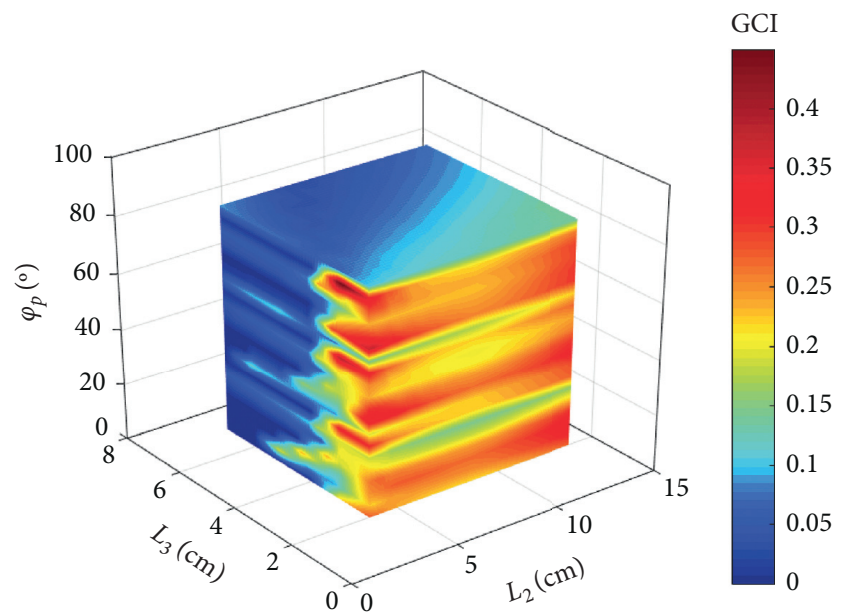

Figure 17: The relationship between the design variables and GCI.

4.5. Optimization Methods and Results Analysis. In this section, the genetic algorithm (GA) is used to analyze the single optimization target and find the optimal results for each performance index. Then, the combined indexes are analyzed comprehensively by a multiobjective genetic algorithm to get the Pareto front.

4.5.1. Optimization Method. The genetic algorithm (GA) is employed for the optimization in this work considering the facts: (1) gradient-based algorithms need not only the value of the objective function but also the derivative of the objective function in the optimization process. However, it is difficult, if not impossible, to differentiate the object function of the case in this work and (2) the GA directly takes the objective function value as the search information.

GA is a method to search for the best solution by simulating the natural evolution process. After the initial population is formed through coding, the task of genetic operation is to impose certain operation on the individuals of the population according to their environmental fitness, so as to realize the evolution process of survival of the fittest.

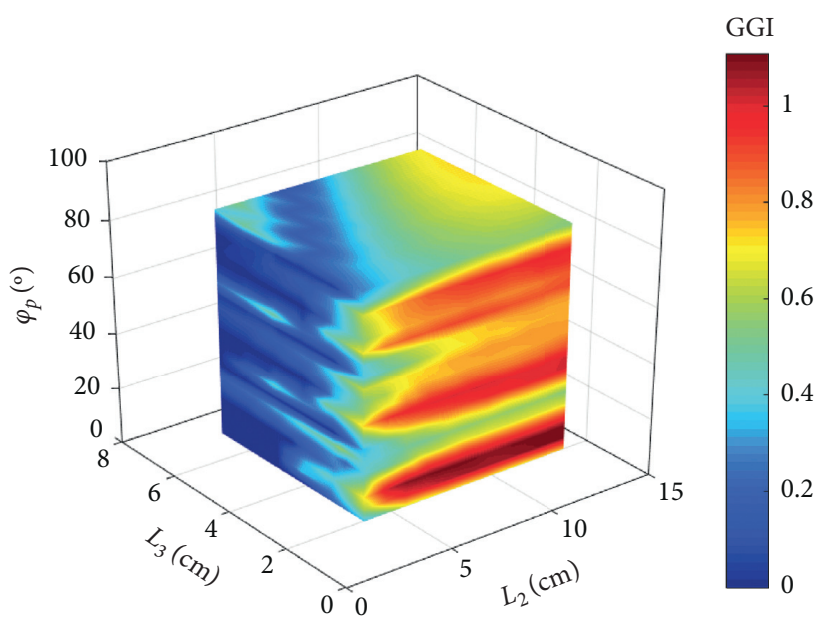

FIgURE 18: The relationship between the design variables and GGI.

From the point of view of optimal search, genetic manipulation can make the solution to the problem be optimized from generation to generation and approximate to the optimal solution. Its main characteristics include the following: (1) there is no derivative and function continuity limit; (2) it has inherent implicit parallelism and better global optimization ability; and (3) the probabilistic optimization method is adopted to adjust the search direction adaptively.

When there are multiple goals, one solution is best on one goal and maybe worst on other goals due to conflicts and incomparability between goals. These solutions while improving any objective function, must weaken the solution of at least one other objective function are called nondominant solutions or Pareto solutions. The set of Pareto solutions is called the Pareto front. All the solutions in the Pareto front are not dominated by the solutions outside the Pareto front. Therefore, these nondominated solutions have the least objective conflict compared with other solutions, which can provide a better choice space for decision-makers.

4.5.2. Single-Objective Optimization. When using GA for single-objective optimization, the parameter settings of GA are shown in Table 3. In the first optimization, the optimization objective function is set as the maximum GWCI. In the second optimization, the optimization objective function is set as the maximum GCI. In the third optimization, the optimization objective function is set as the minimum GGI. Optimization design parameter $X=\left[L_{2}, L_{3}, \varphi_{p}\right]$ must satisfy $L_{2} \in[3,13], L_{3} \in[1.5,6.5], \varphi_{p} \in[5,85]$. The maximum GWCI is 0.6538 and the corresponding design variable $X=$ [12.992, 1.831, 7.418] (Figure 19). The maximum GCI is 0.3076 and the corresponding design variable $X=[12.7,1.5$, 31.586] (Figure 20). The minimum GGI is 0.0306 and the corresponding design variable $X=[7.107,6.484,76.814]$ (Figure 21). In Table 4, the previous value is the result of the corresponding index when $L_{2}=8 \mathrm{~cm}, L_{3}=(10 \sqrt{3} / 3) \mathrm{cm}$, and $\varphi_{p}=45^{\circ}$, which is quite different from the single-objective optimization result, GWCI and GCI are much lower and GGI is much higher. Therefore, single-objective 
TABLE 3: GA parameters.

\begin{tabular}{lc}
\hline Parameter & Setting \\
\hline Population size & 50 \\
Maximum generations & 100 \\
Encoding type & Real \\
Selection strategy & Stochastic sampling \\
Crossover type & Scattered \\
Mutation type & Adaptive \\
\hline
\end{tabular}

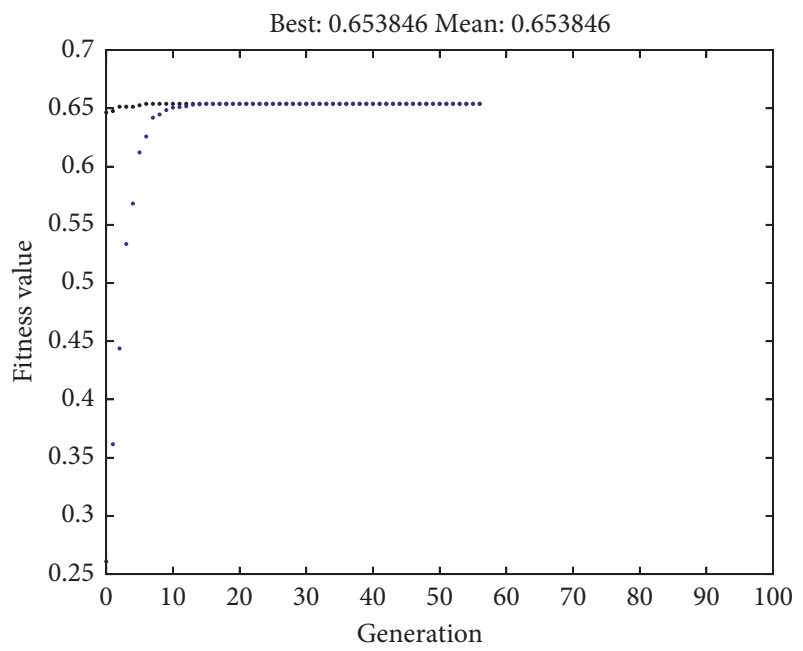

- Best fitness

- Mean fitness

FIGURE 19: GWCI optimization with GA.

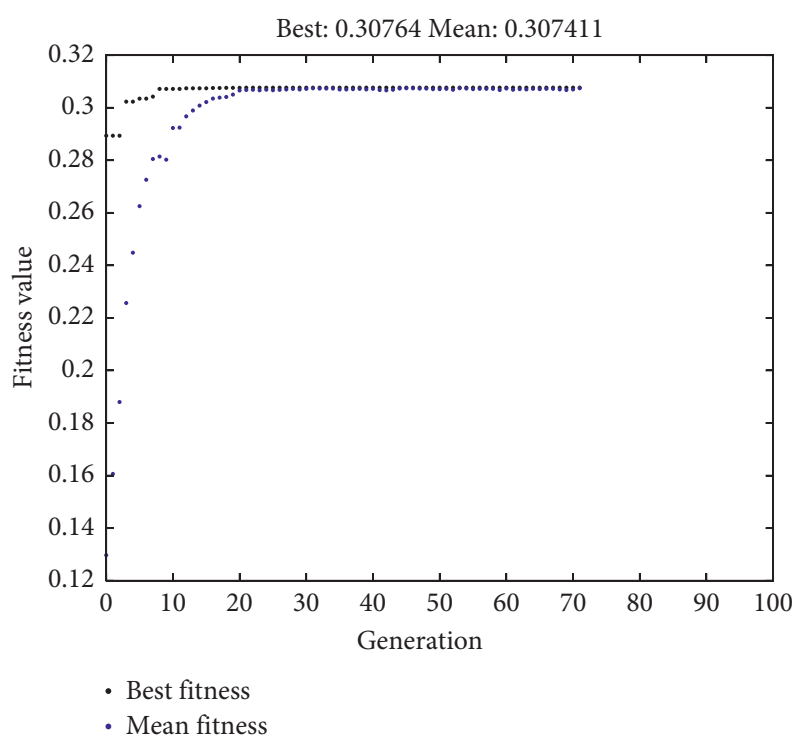

FIgURE 20: GCI optimization with GA.

optimization can achieve the best single performance, but other aspects are not guaranteed.

4.5.3. Multiobjective Optimization Result Analysis. When designing the parameters of the mechanism, it is necessary

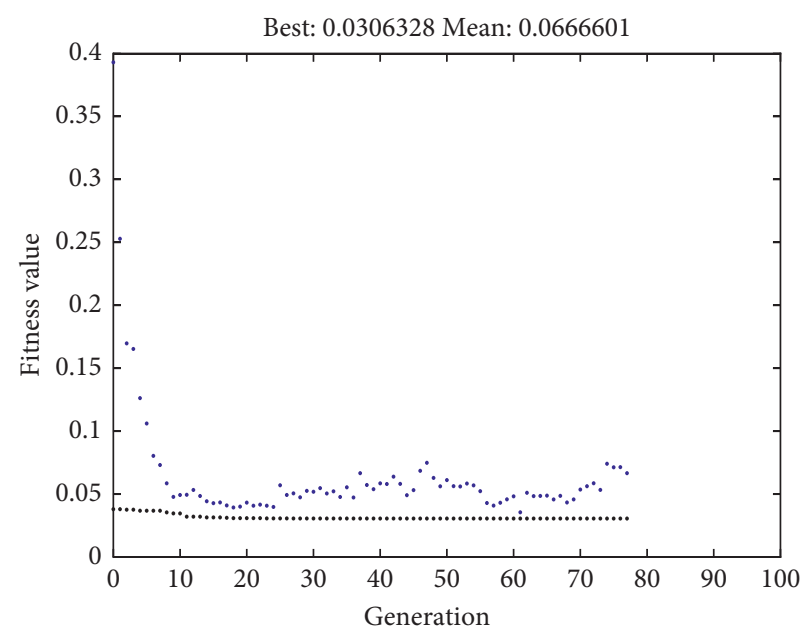

- Best fitness

- Mean fitness

FIGURE 21: GGI optimization with GA.

TABLE 4: Single-objective optimization result.

\begin{tabular}{lccccc}
\hline Index & $L_{2}(\mathrm{~cm})$ & $L_{3}(\mathrm{~cm})$ & $\varphi_{p}($ degree $)$ & Value & Previous value \\
\hline GWCI & 12.992 & 1.831 & 7.418 & 0.6538 & 0.0931 \\
GCI & 12.7 & 1.5 & 31.586 & 0.3076 & 0.0681 \\
GGI & 7.107 & 6.484 & 76.814 & 0.0306 & 0.1368 \\
\hline
\end{tabular}

for the mechanism not only to have a large workspace, but also high sensitivity. At the same time, the sensitivity has a good consistency in the workspace. If only one performance is satisfied and no other performance is considered, the optimization result is not perfect. To sum up, this paper puts forward multiobjective optimization.

The performance evaluation indexes used for optimization are not uniform, and the analysis results vary greatly, and the optimization correlation between different indexes is small, which brings inconvenience to the selection of the optimal design scheme. In this paper, the multiobjective genetic algorithm is used to analyze the three optimization objectives, and the optimization setting parameters are shown in Table 5. The multiobjective optimization problem can be expressed as

$$
\begin{array}{ll}
\max & \{\mathrm{GWCI}, \mathrm{GCI}\} \\
\min & \{\mathrm{GGI}\} \\
\text { over } & X=\left[L_{2}, L_{3}, \varphi_{p}\right] \\
\text { subject to } & L_{2} \in[3,13] \\
& L_{3} \in[1.5,6.5] \\
& \varphi_{p} \in[8,85] .
\end{array}
$$

Through GA multiobjective analysis, the relationship between the three optimization goals of the Pareto optimal solution in the standard space is shown in Figure 22 and the relationship between the two optimization goals is shown in Figures 23-25. The design parameters and optimization 
TABLE 5: GA parameters.

\begin{tabular}{lc}
\hline Parameter & Setting \\
\hline Population size & 40 \\
Maximum generations & 100 \\
Crossover ratio & 1 \\
Pareto fraction & 0.35 \\
Tournament size & 2 \\
Mutation type & Adaptive \\
\hline
\end{tabular}

values corresponding to the Pareto optimal solution are shown in Table 6.

The solution of the multiobjective optimization problem is not unique, but a set of equilibrium solutions, namely, the optimal noninferior solution set or Pareto optimal solution set. Pareto optimality is the ideal state for assigning performance metrics, which means making at least one metric better without making any of them worse. The Pareto optimal solution is only an acceptable solution to the problem. There are 16 Pareto optimal solutions in this optimization problem. According to the requirements of each optimization objective, a solution that meets the design requirements can be selected.

As can be seen from Figures 23-25, GCI increases with the increase of GWCI, GGI increases with the increase of GWCI, and GGI increases with the increase of GCI. However, the evaluation criteria for these three objective functions are different. For parallel manipulator, GWCI and GCI should be large, while GGI should be small. Only in this way can the mechanism have sufficient motion space, flexibility, and motion consistency. Therefore, when designing a planar parallel manipulator, the three performance indexes are equally important. The objective function $\mathrm{GWCI}=0.486$, GCI $=0.145$, and GGI $=0.454$ are selected, and the corresponding design parameters are determined as $L_{2}=11.576 \mathrm{~cm}, L_{3}=2.924 \mathrm{~cm}, \varphi_{p}=42.152^{\circ}$.

\section{Trajectory Optimization}

5.1. Optimization Parameters. Once the structure parameters have been optimized, we can optimize the working area and trajectory of the moving platform to further improve the kinematic and dynamic performance of the presented PPM. The optimization problem involves finding the best area where the motion trajectory of the moving platform should be placed and the point the moving platform should start at the moving trajectory [50].

In this paper, we will evaluate the performance of parallel manipulators under different trajectories. The trajectories take the most common shapes: round, oval, and parabolic. The center position of the moving platform is taken as the optimization variable as

$$
Y=\left[X_{P}, Y_{P}\right] .
$$

First, we analyzed the circle with a radius of $2 \mathrm{~cm} . X_{P}=$ $P_{x 0}+2 \cos (\pi t)$ and $Y_{P}=P_{y 0}+2 \sin (\pi t)$ must be in the workspace for the parallel robot to perform its normal trajectory, $P_{x 0}$ and $P_{y 0}$ are the centers of the moving trajectory. Secondly, we analyzed the ellipse with a long axis of
$4 \mathrm{~cm}$ and a short axis of $2 \mathrm{~cm} . X_{P}=P_{x 0}+2 \cos (\pi t)$ and $Y_{P}=$ $P_{y 0}+\sin (\pi t)$ must be in the workspace for the parallel robot to perform its normal trajectory, $P_{x 0}$ and $P_{y 0}$ are the $X$ and $Y$ coordinates of the center of the ellipse. Finally, we analyzed the trajectory as parabolic. $X_{P}=P_{x 0}+(t-\sin (t))$ and $Y_{P}=$ $P_{y 0}+(1-\cos (t))$ must be in the workspace for the parallel robot to perform its normal trajectory, $P_{x 0}$ and $P_{y 0}$ are the starting point of the parabola. The design variable can be changed to the central position of the trajectory as, which is expressed as

$$
Z=\left[P_{x 0}, P_{y 0}\right] .
$$

According to the calculation in equation (31), the workspace of the planar parallel manipulator is the blue part in Figure 26 and the location of the center of the circle that can satisfy the trajectory in the workspace is the red part in Figure 26.

5.2. Performance Indices. Trajectory optimization is to study the position of trajectory with the best dynamic performance in the process of moving. When the given trajectory is a circle, the working space of the parallel manipulator movement is also determined, so there is no need to consider the global workspace condition index (GWCI). This part mainly studies the sensitivity of the global condition index (GCI) and the global gradient index (GCI) on the moving trajectory and introduces the driving force index (DFI) to find the position of the motion trajectory with the minimum driving force and the maximum sensitivity [51].

5.2.1. GCI and GGI. GCI and GGI have been presented in the previous section. The difference is that the workspace in this part is that of the center position of the moving platform, so the integral in the expression is changed into a line integral $[52,53]$.

$$
\begin{gathered}
\mathrm{GCI}=\frac{\int_{S}(1 / k) \mathrm{d} s}{\int_{S} \mathrm{~d} s}, \\
\mathrm{GGI}=\max _{S}\left\|\frac{\nabla 1}{k(J)}\right\|,
\end{gathered}
$$

where $S$ refers to the track of movement, that is, the circle of radius $2 \mathrm{~cm}$. $k$ is the condition number of the Jacobian matrix. For GCI, the greater the value of the GCI is, the more sensitive the movement process is. For GGI, the smaller the GGI is, the more consistent the sensitivity becomes during the movement. Figure 27 shows the relationship between the center of the track and the GCI. Figure 28 shows the relationship between the center of the trajectory and the GGI.

5.2.2. Driving Force Index (DFI). Dynamic mechanical force is not only an important parameter that affects the kinematics and dynamic behaviors of the robot manipulator, but also an important basis for the design of mechanical strength and the shape of structure $[54,55]$. Therefore, mechanical 


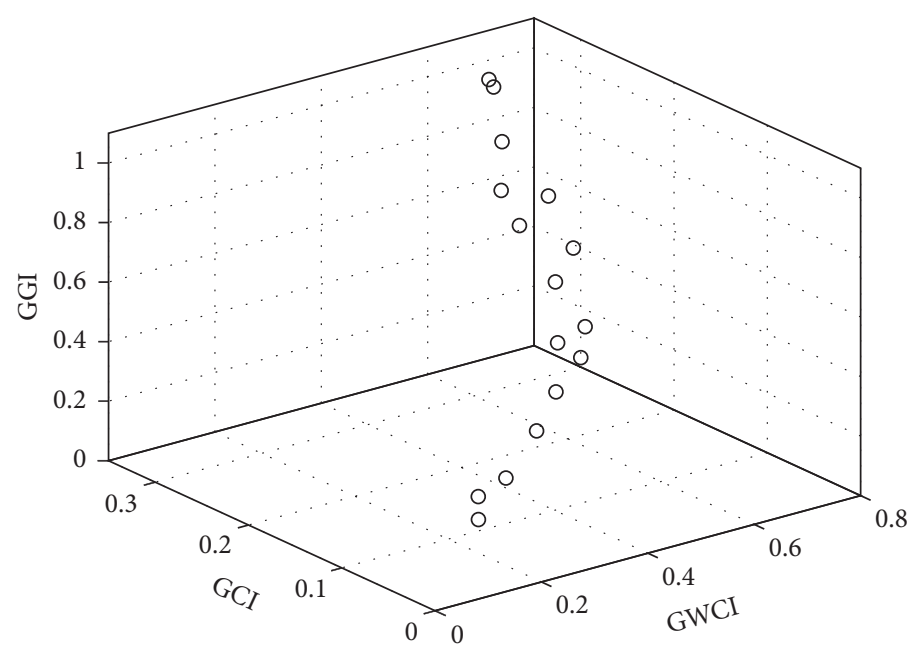

Figure 22: Pareto front for GWCI, GCI, GGI.

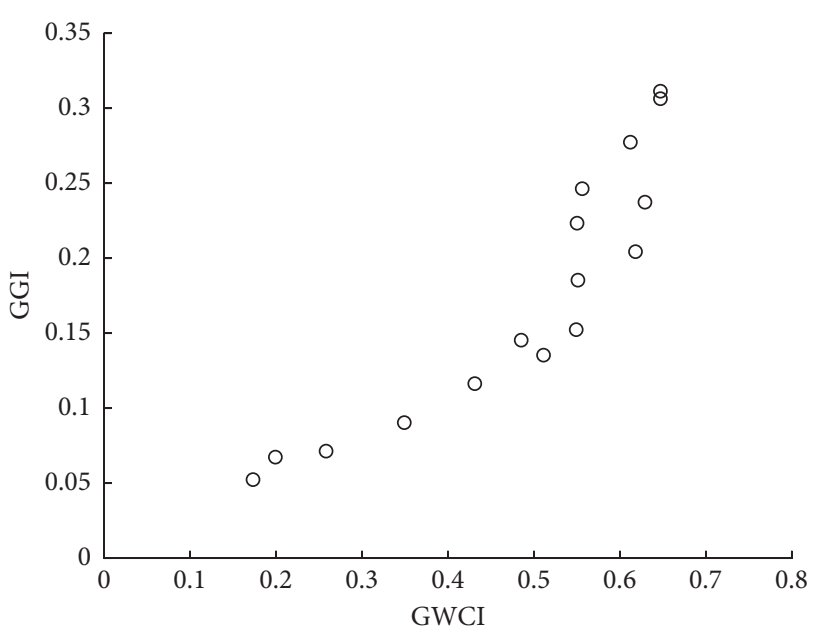

FIgUre 23: Pareto front for GWCI, GCI.

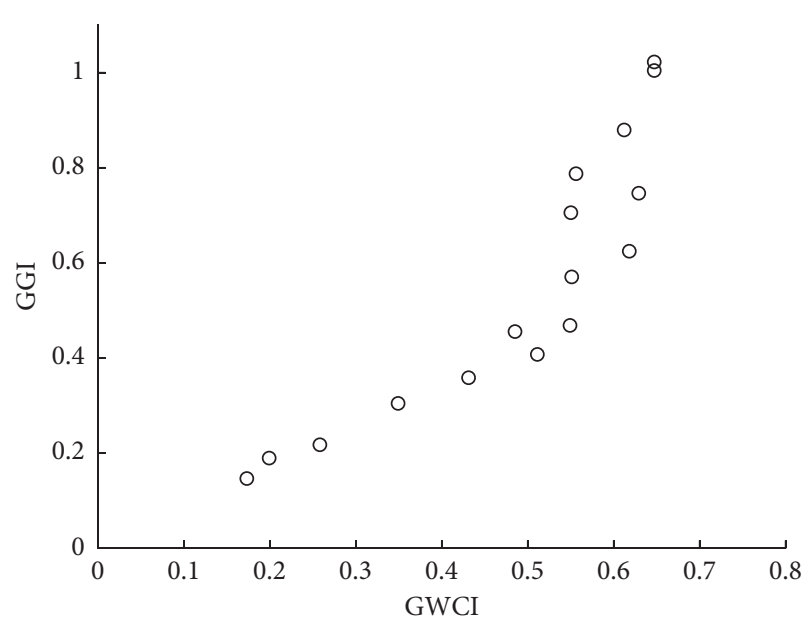

FIgUre 24: Pareto front for GWCI, GGI.

force must be analyzed when designing new mechanical equipment or optimizing existing robot manipulators. For the planar parallel manipulator, the driving force of the

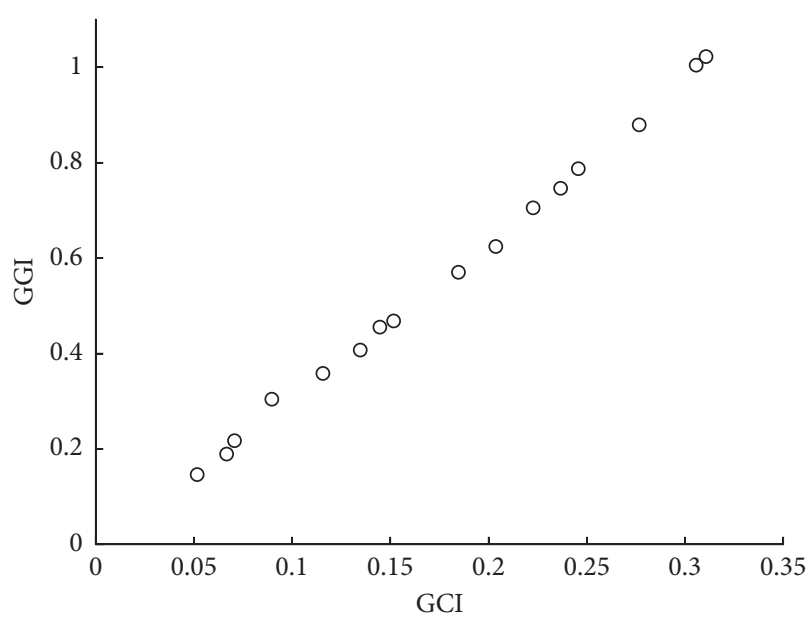

FIgURE 25: Pareto front for GCI, GGI.

motor is mainly considered. In order to study the driving force, we propose the Driving Force Index (DFI), which is the maximum driving force required by moving the platform in a given motion, as the optimization objective.

$$
f(x, y)=\max |F(X p, Y p)|
$$

where $X p$ and $Y p$ are the movement trajectory within $t=4 \mathrm{~s}$, and $F$ can be obtained from equation (29). The maximum driving force required in the three branch chains is obtained as the optimization index. The smaller the driving force is, the better the mechanical performance will be. Figure 29 shows the DFI performance graph of the planar parallel robot moving platform.

5.3. Optimization Results and Analysis. Optimization of the dynamic parameters can make the moving platform have better motion performance in the process of movement. In the optimization process, the main consideration is the value of each performance index on the movement trajectory [56]. As can be seen from Figure 27, the trajectory center changes 
TABle 6: Pareto optimal solutions.

\begin{tabular}{|c|c|c|c|c|c|c|}
\hline Index & GWCI & GCI & GGI & $L_{2}$ & $L_{3}$ & $\varphi_{p}$ \\
\hline 1 & 0.648 & 0.306 & 1.003 & 12.992 & 1.534 & 33.671 \\
\hline 2 & 0.648 & 0.311 & 1.021 & 12.992 & 1.503 & 33.546 \\
\hline 3 & 0.552 & 0.185 & 0.569 & 12.21 & 2.399 & 42.342 \\
\hline 4 & 0.613 & 0.277 & 0.878 & 12.781 & 1.698 & 36.196 \\
\hline 5 & 0.432 & 0.116 & 0.357 & 11.382 & 3.428 & 51.999 \\
\hline 6 & 0.259 & 0.071 & 0.216 & 9.653 & 4.98 & 42.374 \\
\hline 7 & 0.35 & 0.09 & 0.303 & 10.241 & 4.416 & 34.335 \\
\hline 8 & 0.2 & 0.067 & 0.188 & 8.984 & 5.335 & 39.583 \\
\hline 9 & 0.174 & 0.052 & 0.145 & 8.878 & 6.465 & 36.601 \\
\hline 10 & 0.63 & 0.237 & 0.745 & 12.945 & 2.064 & 35.399 \\
\hline 11 & 0.619 & 0.204 & 0.623 & 12.852 & 2.264 & 41.232 \\
\hline 12 & 0.512 & 0.135 & 0.406 & 12.104 & 3.203 & 44.159 \\
\hline 13 & 0.55 & 0.152 & 0.467 & 12.192 & 2.829 & 44.308 \\
\hline 14 & 0.557 & 0.246 & 0.786 & 12.091 & 1.882 & 35.559 \\
\hline 15 & 0.551 & 0.223 & 0.704 & 12.007 & 2.044 & 37.38 \\
\hline 16 & 0.486 & 0.145 & 0.454 & 11.576 & 2.924 & 42.152 \\
\hline
\end{tabular}

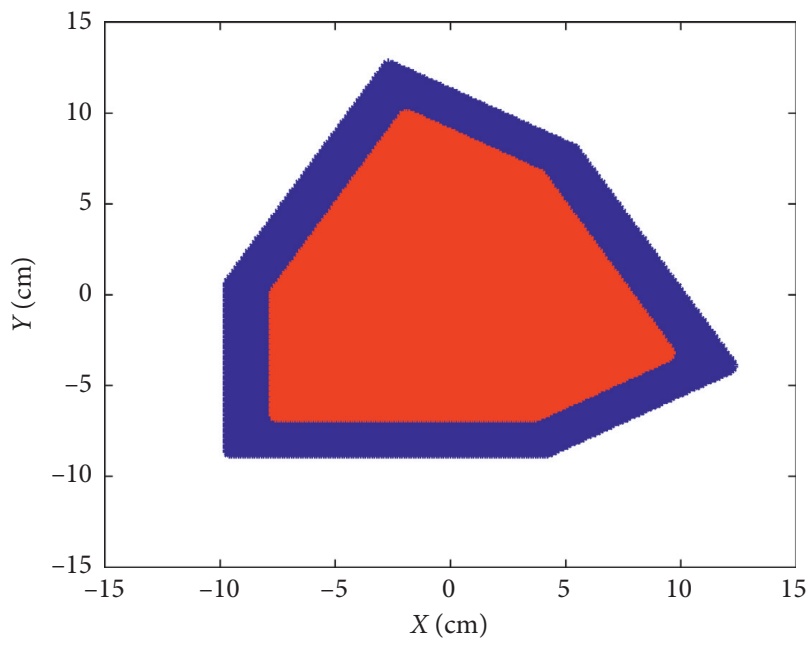

(a)

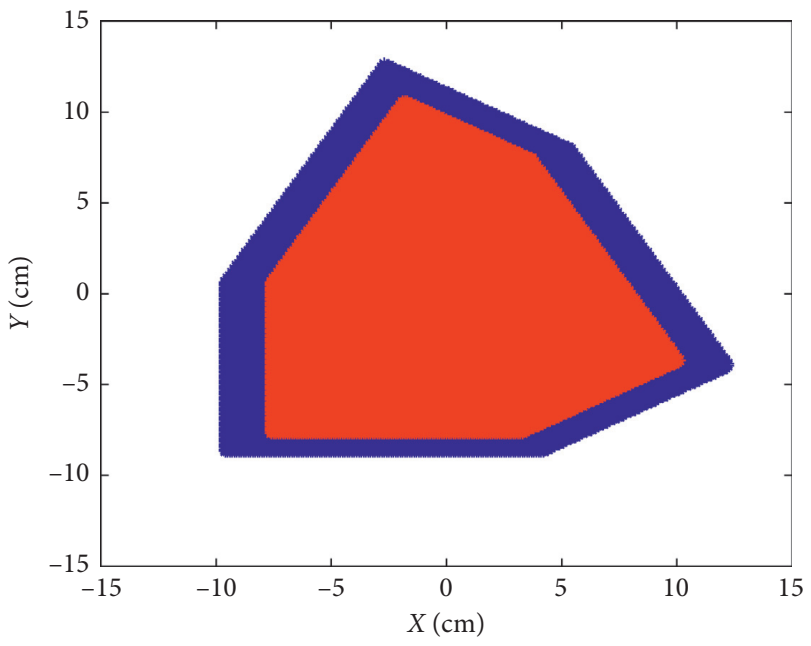

(b)

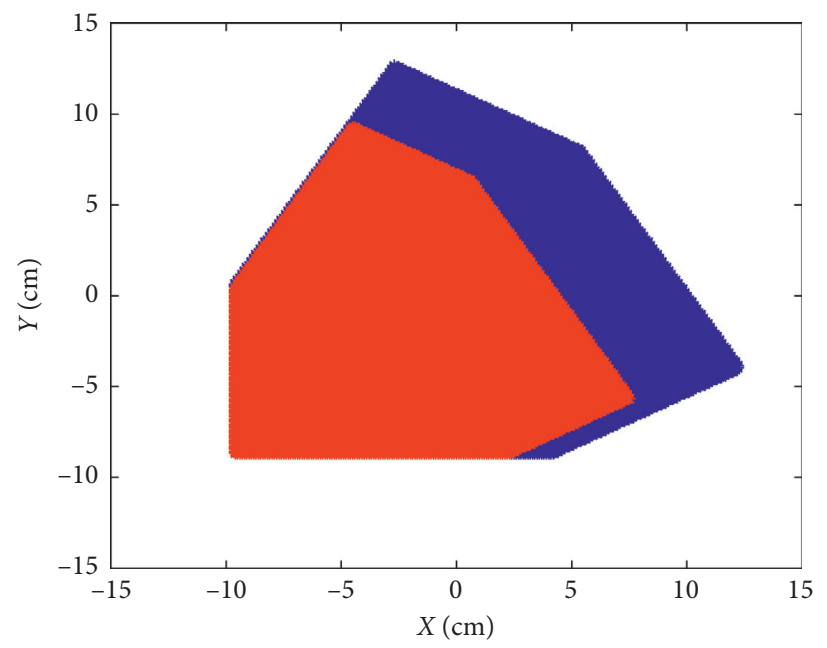

(c)

Figure 26: The workspace. (a) The circle trajectory, (b) the ellipse trajectory, and (c) the parabolic trajectory. 


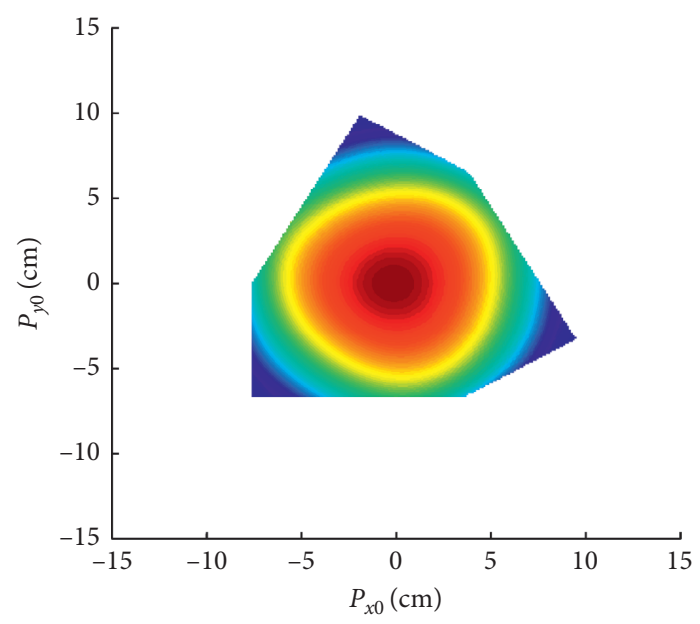

(a)

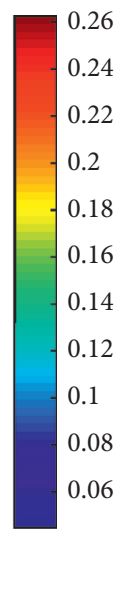

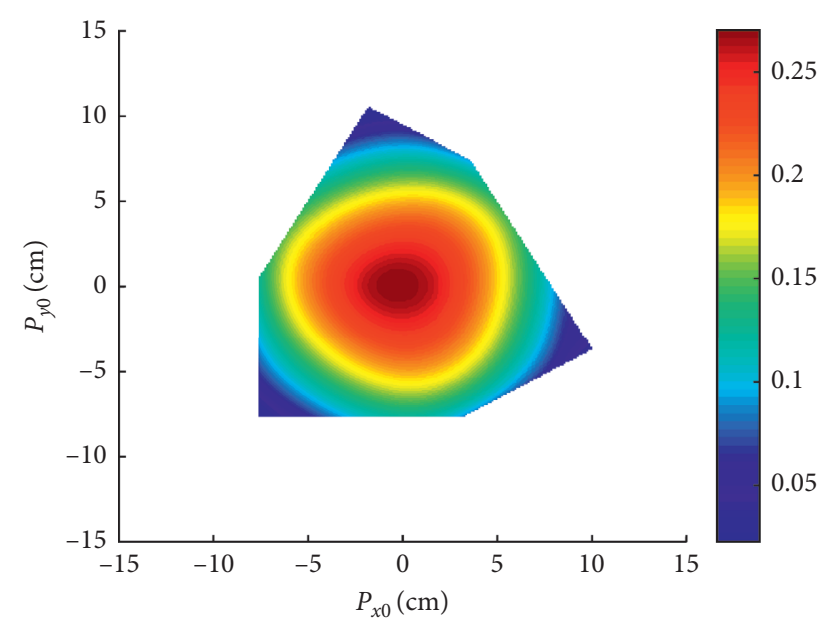

(b)

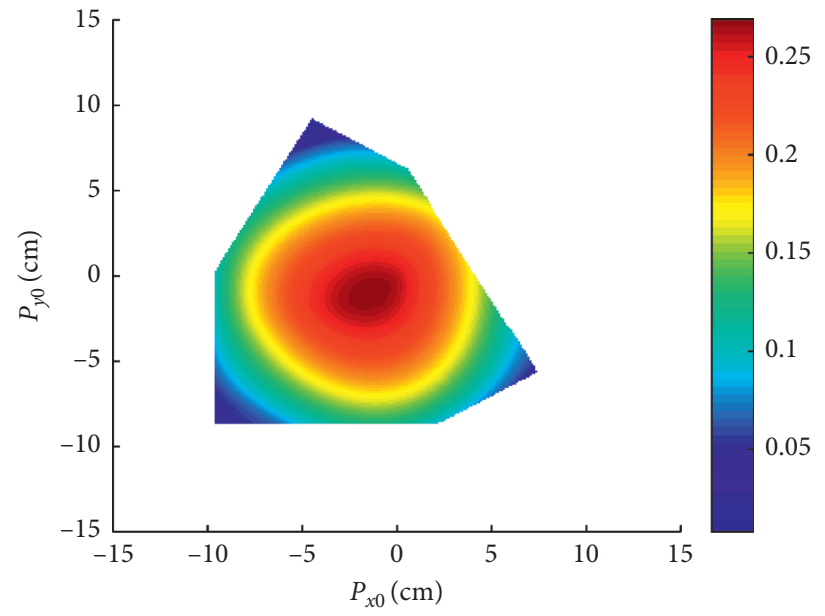

(c)

FIgURE 27: Relationship between GCI and $P_{x 0}, P_{y 0}$. (a) The circle trajectory, (b) the ellipse trajectory, and (c) the parabolic trajectory.

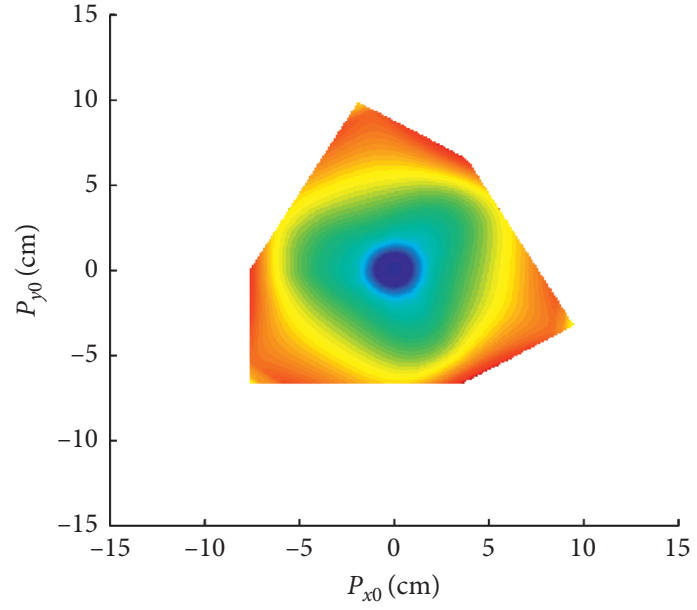

(a)

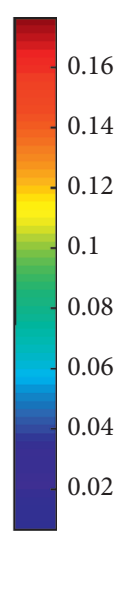

Figure 28: Continued.

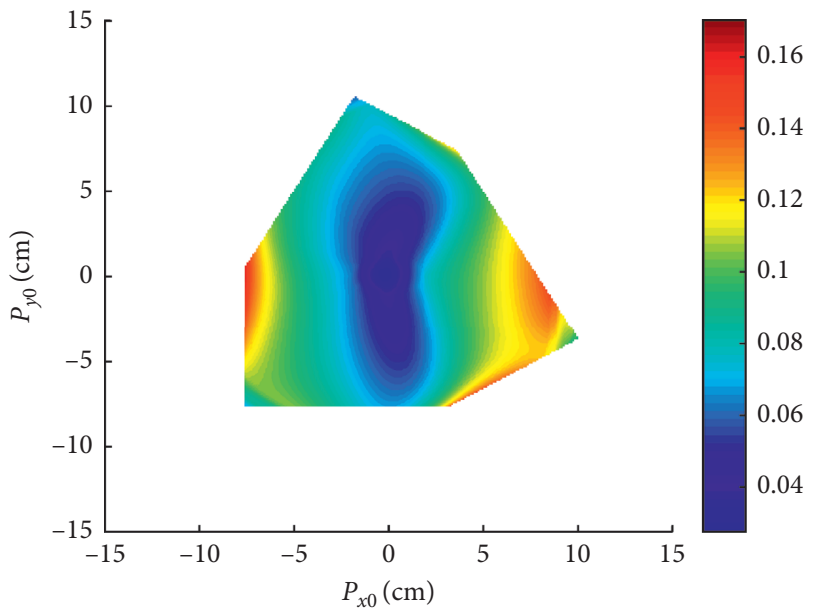

(b) 


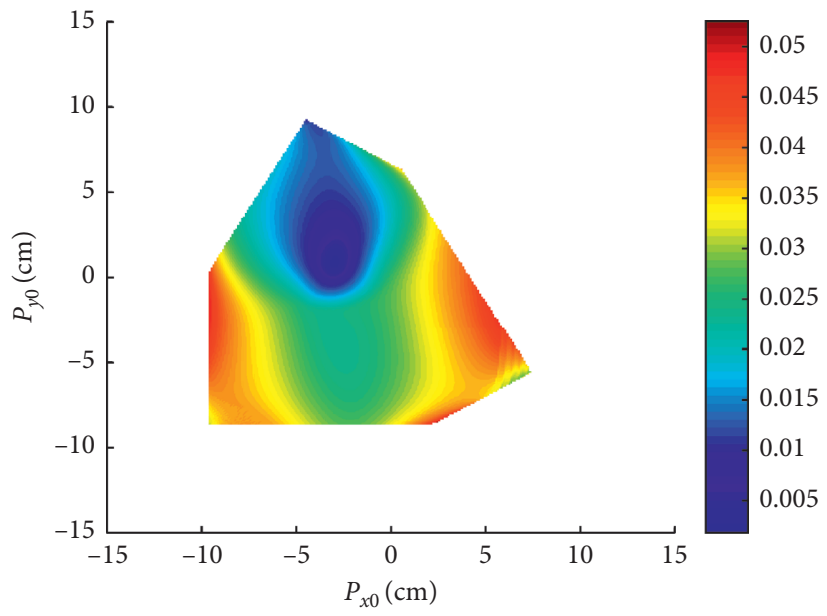

(c)

FIGURE 28: Relationship between GGI and $P_{x 0}, P_{y 0}$. (a) The circle trajectory, (b) the ellipse trajectory, and (c) the parabolic trajectory.

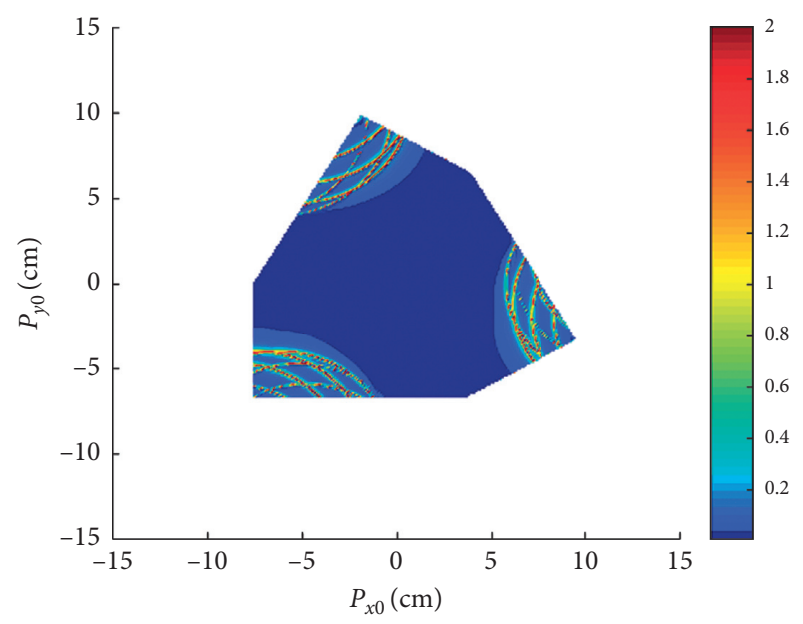

(a)

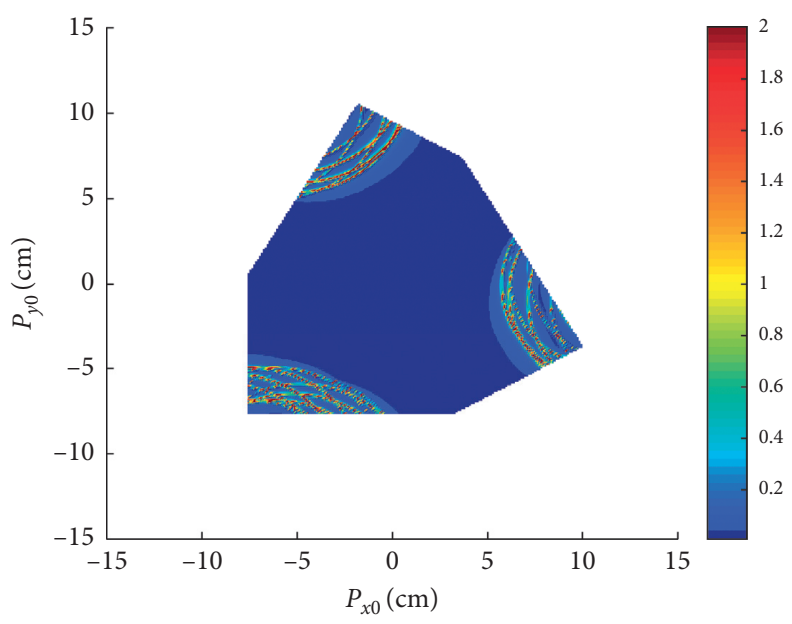

(b)

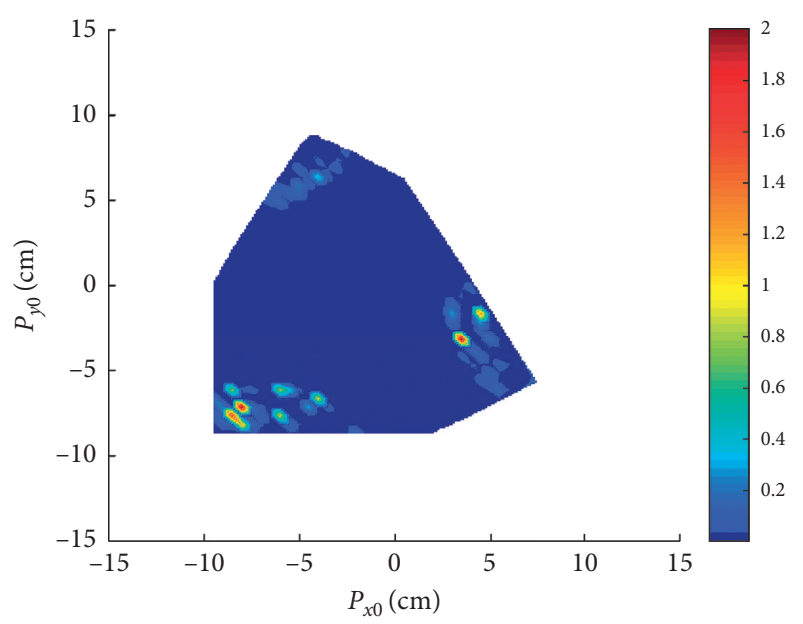

(c)

FIgURe 29: Relationship between DFI and $P_{x 0}, P_{y 0}$. (a) The circle trajectory, (b) the ellipse trajectory, and (c) the parabolic trajectory. 


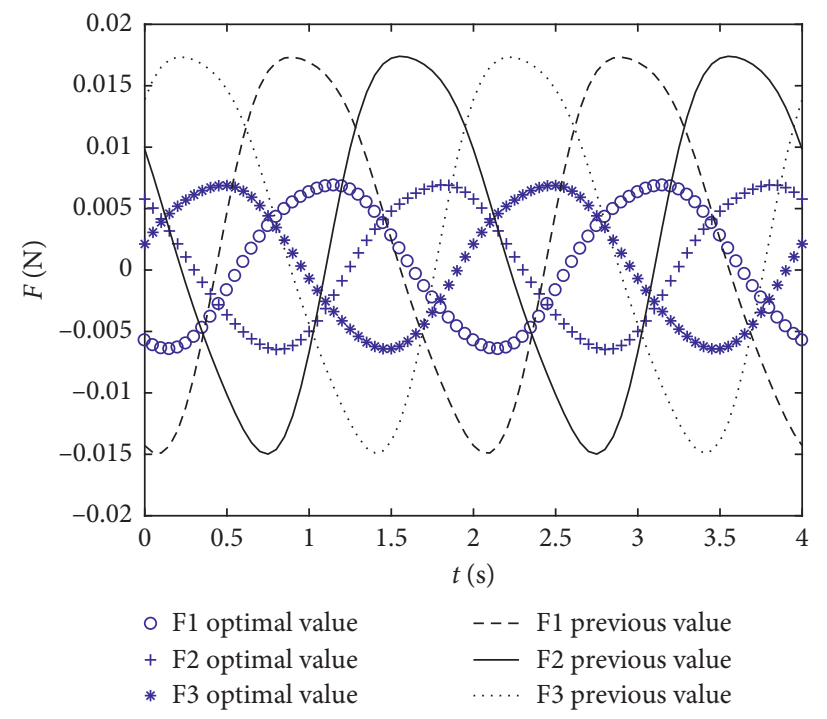

Figure 30: Comparison of driving forces before and after structural optimization.

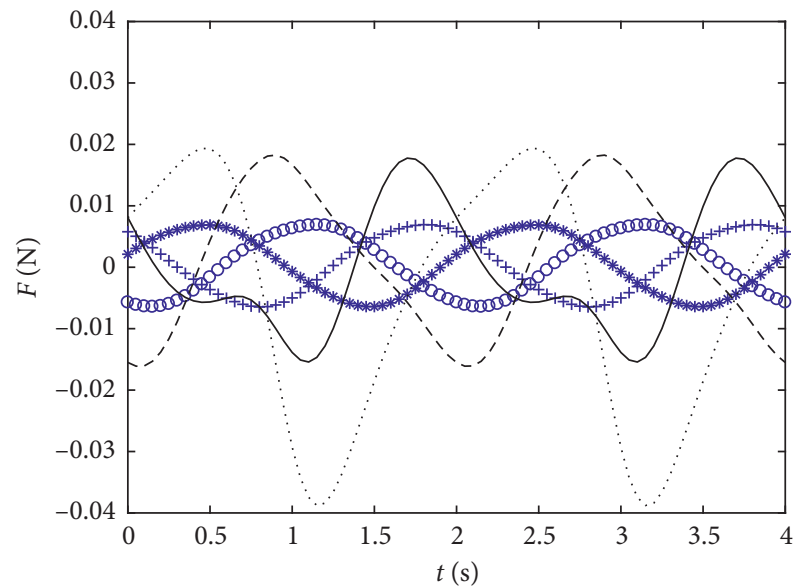

F1 include the dynamic index

$+\mathrm{F} 2$ include the dynamic index

* F3 include the dynamic index

(a) the dynamic index F3 exclude the dynamic index

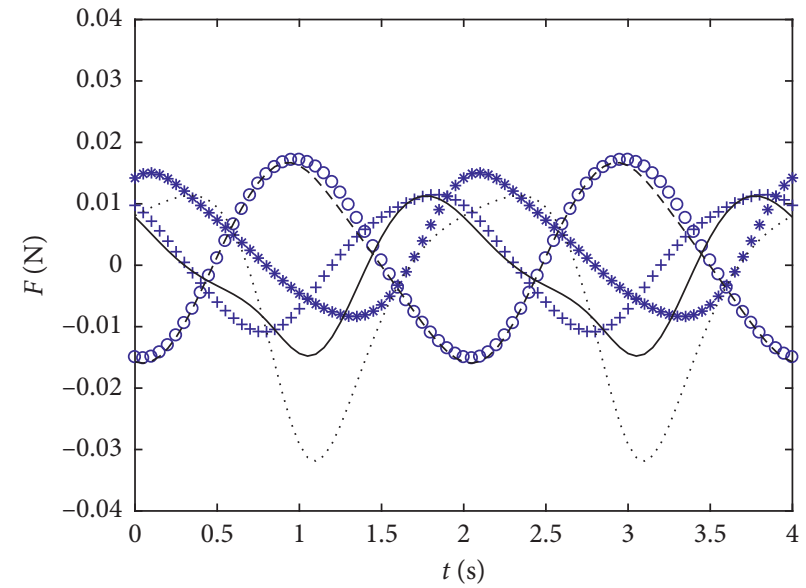

- F1 include the dynamic index

$+\mathrm{F} 2$ include the dynamic index

* F3 include the dynamic index

-. - F1 exclude the dynamic index

- F2 exclude the dynamic index
.... F3 exclude the dynamic index

(b)

Figure 31: Continued. 


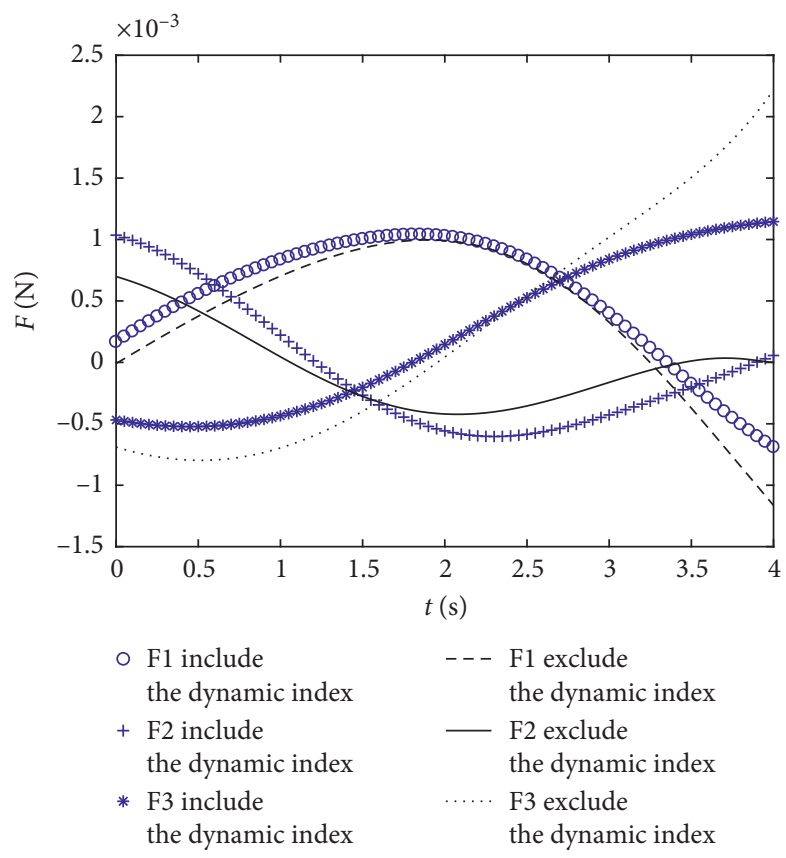

(c)

Figure 31: Comparison of driving forces before and after using dynamic indicators. (a) The circle trajectory, (b) the ellipse trajectory, and (c) the parabolic trajectory.

from the origin of coordinates to the edge of the workspace, and the sensitivity of the planar parallel manipulator in the process of motion becomes smaller and smaller. As can be seen from Figure 28, the sensitivity of the trajectory center of the moving platform in the blue area is very consistent, and the mechanical platform is relatively stable in the process of movement. As can be seen from Figure 29, the closer the position is to the center of the coordinate system, the smaller the maximum driving force is required, which can reduce the size or the energy consumption of the motor. Considering the three indexes of GCI, GGI, and DFI, the center position of the motion trajectory is selected at $(0,0)$, which has the optimal kinematic and dynamic performance.

\section{Discussion and Conclusion}

With the proposed methodology and procedure of optimizing the structure size and motion trajectory of the parallel planar manipulator, the robot manipulator can achieve optimal kinematic and dynamic performance for its applications. Through the optimization, the optimal parameters are obtained as $L_{2}=11.576 \mathrm{~cm}, L_{3}=2.924 \mathrm{~cm}$, $\varphi_{p}=42.152, P_{x 0}=0$, and $P_{y 0}=0$. It can be seen that the dynamic performance has been greatly improved after optimization, no matter under which moving trajectory, and the planar parallel manipulator only needs a small force to generate the same motion trajectory while keeping high kinematic performance. The maximum driving force required is $0.0174 \mathrm{~N}$ before the optimization in the circle trajectory and is significantly reduced to be $0.0069 \mathrm{~N}$ after the optimization (Figure 30). In different trajectories, the dynamic performance is improved by using the dynamic index (Figure 31). The root mean square error is used to measure the deviation between the preoptimization value and the postoptimization value. Since the structural optimization does not involve the dynamics index when calculating the driving force's root mean square error, this paper only enumerates the driving force for the slider in the circular trajectory. In trajectory optimization, dynamic index is involved. In order to highlight the importance of this index, this paper calculates the root mean square error of circular trajectory, ellipse trajectory, and parabolic trajectory, respectively. Under different trajectories (Table 7), some driving forces will have large errors after dynamic optimization, such as F3 of circular trajectory, which shows the importance and effectiveness of using dynamic indexes in robot optimization.

The planar parallel manipulator has been widely used as kinematics and dynamic machines, but it has two main disadvantages: complex dynamic equations, limited workspace, and singularity. In order to improve these two shortcomings, the discrete time transfer matrix method (DT-TMM) has been proposed to improve the computational efficiency and structure optimization and trajectory optimization have been conducted to improve the workspace and avoid generating singular configuration. In order to optimize the structure size of the 3-PRR planar parallel manipulator, GWCI, GCI, and GGI have been taken as optimization objectives, GA is used to analyze a single target, and multiobjective genetic algorithm is used to find Pareto optimal solution. In order to improve the dynamic performance of the manipulator, the driving 
TABLE 7: The root mean square error between the difference cases.

\begin{tabular}{|c|c|c|c|c|}
\hline \multicolumn{2}{|l|}{ Cases } & F1 & $\mathrm{F} 2$ & F3 \\
\hline \multicolumn{2}{|c|}{ Before and after structural optimization } & 0.0077 & 0.0077 & 0.0077 \\
\hline \multirow{3}{*}{ Before and after using dynamic indicators } & The circle trajectory & 0.0076 & 0.0066 & 0.0168 \\
\hline & The ellipse trajectory & 0.0012 & 0.0051 & 0.0113 \\
\hline & The parabolic trajectory & $1.6054 e-04$ & $2.1375 e-04$ & $3.3516 e-04$ \\
\hline
\end{tabular}

force index is proposed and integrated into the optimization target, and the optimal moving trajectory has been obtained by combining the kinematic indices. The two major contributions of this paper to the design and control of parallel robot manipulators can be summarized as follows: (1) extending DT-TMM to establishing the dynamic model with high computation efficiency, and (2) proposing a new dynamic index and integrating the dynamic index into the dynamic design and motion optimization with the developed dynamic modeling method and the GA optimization technique. The developed modeling method, and design and motion optimization strategies have been demonstrated and verified with a 3PRR parallel manipulator, and can be extended to the dynamic modeling, and optimization design for general parallel manipulators with flexible components.

\section{Data Availability}

The data used to support the findings of this study are available from the corresponding author upon request.

\section{Disclosure}

The preliminary results of this work were presented in proceedings of " 15 th International Conference on Multibody Systems, Nonlinear Dynamics, and Control" titled "Dynamic Modelling of a Planar Parallel Robot Manipulator Using the Discrete Time Transfer Matrix Method."

\section{Conflicts of Interest}

The authors declare that they have no conflicts of interest.

\section{References}

[1] Y. Zhang, Y. Dang, and X. Wu, "Kinematics and singularity analysis of a novel decoupled translational parallel manipulator," Mechanical Science \& Technology for Aerospace Engineering, vol. 27, no. 4, pp. 622-626, 2010.

[2] Q. Zhang, X. Zhang, and J. Liang, "Dynamic analysis of planar 3-RRR flexible parallel robot," in Proceedings of the IEEE International Conference on Robotics \& Biomimetics, IEEE, Guangzhou, China, December 2012.

[3] B. S. Kim, "Dynamic and kinematic analysis of 6-bar parallel robot," in Proceedings of the 2013 44th International Symposium on IEEE, Seoul, South Korea, 2013.

[4] G. Bogdan, P. Doina, V. Calin, and N. Plitea, "Development of inverse dynamic model for a surgical hybrid parallel robot with equivalent lumped masses," Robotics and ComputerIntegrated Manufacturing, vol. 28, no. 3, pp. 402-415, 2012.

[5] A. M. Hay and J. A. Snyman, "Methodologies for the optimal design of parallel manipulators," International Journal for
Numerical Methods in Engineering, vol. 59, no. 11, pp. 131152, 2004.

[6] Q. Zhang, J. K. Mills, W. L. Cleghorn, J. Jin, and C. Zhao, "Trajectory tracking and vibration suppression of a 3-PRR parallel manipulator with flexible links," Multibody System Dynamics, vol. 33, no. 1, pp. 27-60, 2015.

[7] D. Liang, Y. Song, T. Sun, and X. Jin, "Rigid-flexible coupling dynamic modeling and investigation of a redundantly actuated parallel manipulator with multiple actuation modes," Journal of Sound and Vibration, vol. 403, pp. 129-151, 2017.

[8] R. W. Krauss, "Computationally efficient modeling of flexible robots using the transfer matrix method," Journal of Vibration and Control, vol. 18, no. 5, pp. 596-608, 2012.

[9] X. Zhang, R. Sørensen, M. R. Iversen, and H. Li, "Computationally efficient dynamic modeling of robot manipulators with multiple flexible-links using acceleration-based discrete time transfer matrix method," Robotics and Computer-Integrated Manufacturing, vol. 49, pp. 181-193, 2018.

[10] B. He, X. Rui, and G. Wang, "Riccati discrete time transfer matrix method for elastic beam undergoing large overall motion," Multibody System Dynamics, vol. 18, no. 4, pp. 579-598, 2007.

[11] X. Rui, D. Bestle, J. Zhang, and Q. Zhou, "A new version of transfer matrix method for multibody systems," Multibody System Dynamics, vol. 38, no. 2, pp. 137-156, 2016.

[12] X. Rui, X. Wang, Q. Zhou, and J. Zhang, “Transfer matrix method for multibody systems (Rui method) and its applications," Science China Technological Sciences, vol. 62, no. 5, pp. 712-720, 2019.

[13] X. Rui, G. Wang, and J. Zhang, Transfer Matrix Method for Multibody Systems: Theory and Applications, Wiley, Hoboken, NJ, USA, 2018.

[14] X. Rui, G. Wang, and L. Yun, "Study on the vibration characteristics of LRMLRS," Journal of Vibration \& Shock, vol. 24, no. 1, pp. 8-12, 2005.

[15] B. Rong, X. Rui, and L. Tao, "Discrete time transfer matrix method for launch dynamics modeling and cosimulation of self-propelled artillery system," Journal of Applied Mechanics, vol. 80, no. 1, 2013.

[16] X. Rui, G. Wang, Y. Lu, and L. Yun, "Transfer matrix method for linear multibody system," Multibody System Dynamics, vol. 19, no. 3, pp. 179-207, 2008.

[17] R. Krauss and M. Okasha, "Discrete-time transfer matrix modeling of flexible robots under feedback control," in Proceedings of the American Control Conference (ACC), pp. 4104-4109, Washington, DC, USA, June 2013.

[18] Q. Zhang, C. Li, and J. Zhang, "Synchronized motion control and precision positioning compensation of a 3-DOFs macromicro parallel manipulator fully actuated by piezoelectric actuators," Smart Materials and Structures, vol. 26, pp. 1-15, 2017.

[19] Z. Zhou, J. Xi, and C. K. Mechefske, "Modeling of a fully flexible 3PRS manipulator for vibration analysis," Journal of Mechanical Design, vol. 128, no. 2, pp. 403-412, 2006. 
[20] A. Kumar and K. J. Waldron, "The workspaces of a mechanical manipulator," Journal of Mechanical Design, vol. 103, no. 3, pp. 665-672, 1981.

[21] R. Koeppe and T. Yoshikawa, "Dynamic manipulability analysis of compliant motion," in Proceedings of the IEEE/RSJ International Conference on Intelligent Robots \& Systems, pp. 1472-1478, San Diego, CA USA, December 1997.

[22] C. Melchiorri and J. K. Salisbury, "An algorithm for the control of a hand-arm robotic system," in Proceedings of the Fifth International Conference on Advanced Robotics, pp. 471-476, Pisa, Italy, June 1991.

[23] C. Gosselin and J. Angeles, "A global performance index for the kinematic optimization of robotic manipulators," Journal of Mechanical Design, vol. 113, no. 3, pp. 220-226, 1991.

[24] R. E. Stamper, L.-W. Tsai, and G. C. Walsh, "Optimization of a three DOF translational platform for well-conditioned workspace," in Proceedings of the IEEE International Conference on Robotics and Automation, pp. 3250-3255, Albuquerque, NM, USA, April 1997.

[25] E. Courteille, D. Deblaise, and P. Maurine, "Design optimization of a delta-like parallel robot through global stiffness performance evaluation," in Proceedings of the IEEE/RSJ International Conference on Intelligent Robots \& Systems, pp. 5159-5166, St Louis, MI, USA, October 2009.

[26] R. Kurazume and T. Hasegawa, "A new index of serial-link manipulator performance combining dynamic manipulability and manipulating force ellipsoids," IEEE Transactions on Robotics, vol. 22, no. 5, pp. 1022-1028, 2006.

[27] V. Rezania and S. Ebrahimi, "Dexterity characterization of the RPR parallel manipulator based on the local and global condition indices," Journal of Mechanical Science and Technology, vol. 31, no. 1, pp. 335-344, 2017.

[28] S. Bi, Z. Zhuang, and T. Xia, "Multi-objective optimization for a humanoid robot walking on slopes," in Proceedings of the International Conference on Machine Learning \& Cybernetics, pp. 1261-1267, Kuantan, Malaysia, 2011.

[29] J. Luo, D. Wang, Z. Xing et al., "Multi-objective optimal kinematic design of 3-TPS/TP parallel robot manipulator," Advanced Engineering Forum, vol. 2012, no. 2-3, pp. 324-329, 2012.

[30] J. Gallardo-Alvarado and J. H. Tinajero-Campos, "A parallel manipulator with planar configurable platform and three endeffectors," Mathematical Problems in Engineering, vol. 2019, Article ID 7972837, 12 pages, 2019.

[31] J. Mo, Z. Qiu, L. Zeng, and X.-M. Zhang, "A new calibration method for a directly driven 3PRR positioning system," Journal of Intelligent \& Robotic Systems, vol. 85, no. 3-4, pp. 613-631, 2017.

[32] R. Chandra, M. Zhang, and L. Mengjie, "Solving the forward kinematics of the 3RPR planar parallel manipulator using a hybrid meta-heuristic paradigm," in Proceedings of the International Symposium on Computational Intelligence in Robotics and Automation, pp. 177-182, Daejeon, Republic of Korea, December 2009.

[33] L. Baron and J. Angeles, "The direct kinematics of parallel manipulators under joint-sensor redundancy," IEEE Transactions on Robotics and Automation, vol. 16, no. 1, pp. 12-19, 2000.

[34] G. Chen, X. Rui, L. K. Abbas, G. Wang, F. Yang, and W. Zhu, "A novel method for the dynamic modeling of Stewart parallel mechanism," Mechanism and Machine Theory, vol. 126, no. 8, pp. 397-412, 2018.

[35] E. Macho, O. Altuzarra, E. Amezua, and A. Hernandez, "Obtaining configuration space and singularity maps for parallel manipulators," Mechanism and Machine Theory, vol. 44, no. 11, pp. 2110-2125, 2009.

[36] I. A. Bonev and J. Ryu, "A geometrical method for computing the constant-orientation workspace of 6-PRRS parallel manipulators," Mechanism and Machine Theory, vol. 36, no. 1, pp. 1-13, 2001.

[37] B. Monsarrat and C. M. Gosselin, "Workspace analysis and optimal design of a 3-leg 6-DOF parallel platform mechanism," IEEE Transactions on Robotics and Automation, vol. 19, no. 6, pp. 954-966, 2003.

[38] C. B. Pham, S. H. Yeo, and G. Yang, "Workspace analysis and optimal design of cable-driven planar parallel manipulators," in Proceedings of the IEEE Conference on Robotics, Automation and Mechatronics, pp. 219-224, Singapore, December 2004.

[39] I. Ebrahimi, J. A. Carretero, and R. Boudreau, "3-PRRR redundant planar parallel manipulator: inverse displacement, workspace and singularity analyses," Mechanism and Machine Theory, vol. 42, no. 8, pp. 1007-1016, 2007.

[40] C. Brisan and A. Csiszar, "Computation and analysis of the workspace of a reconfigurable parallel robotic system," Mechanism and Machine Theory, vol. 46, no. 11, pp. 16471668, 2011.

[41] J. Wang, J. Wu, T. Li, and X. Liu, "Workspace and singularity analysis of a 3-DOF planar parallel manipulator with actuation redundancy," Robotica, vol. 27, no. 1, pp. 51-57, 2009.

[42] C.-T. Chen and H.-W. Chi, "Singularity-free trajectory planning of platform-type parallel manipulators for minimum actuating effort and reactions," Robotica, vol. 26, no. 3, pp. 371-384, 2008.

[43] J. Sefrioui and C. M. Gosselin, "Singularity analysis and representation of planar parallel manipulators," Robotics and Autonomous Systems, vol. 10, no. 4, pp. 209-224, 1992.

[44] J. Mo, X. Zhang, and Z. Qiu, "Control strategy research on avoid and escape the singular area of the 3PRR parallel platform based on SEM environment," Journal of Mechanical Engineering, vol. 51, no. 23, pp. 1-11, 2015.

[45] S. Kucuk and Z. Bingul, "Comparative study of performance indices for fundamental robot manipulators," Robotics and Autonomous Systems, vol. 54, no. 7, pp. 567-573, 2006.

[46] J. Enferadi and R. Nikrooz, "The performance indices optimization of a symmetrical fully spherical parallel mechanism for dimensional synthesis," Journal of Intelligent \& Robotic Systems, vol. 90, no. 3-4, pp. 305-321, 2018.

[47] C. Gosselin and J. Angeles, "The optimum kinematic design of a planar three-degree-of-freedom parallel manipulator," Journal of Mechanisms, Transmissions, and Automation in Design, vol. 110, no. 1, pp. 35-41, 1988.

[48] C. M. Gosselin and E. Lavoie, "On the kinematic design of spherical three-degree-of- freedom parallel manipulators," The International Journal of Robotics Research, vol. 12, no. 4, pp. 394-402, 1993.

[49] R. Kurtz and V. Hayward, "Multiple-goal kinematic optimization of a parallel spherical mechanism with actuator redundancy," IEEE Transactions on Robotics and Automation, vol. 8, no. 5, pp. 644-651, 1992.

[50] G. Boschetti, R. Rosa, and A. Trevisani, "Optimal robot positioning using task-dependent and direction-selective performance indexes: general definitions and application to a parallel robot," Robotics and Computer-Integrated Manufacturing, vol. 29, no. 2, pp. 431-443, 2013.

[51] S. Kucuk and Z. Bingul, "Robot workspace optimization based on a novel local and global performance indices," in Proceedings of the IEEE International Symposium on Industrial 
Electronics, vol. 4, pp. 1593-1958, Dubrovnik, Croatia, June 2005.

[52] F. A. Laramolina, J. M. Rosario, and D. Dumur, "Multi-objective design of parallel manipulator using global indices," The Open Mechanical Engineering Journal, vol. 4, no. 1, pp. 37-47, 2010.

[53] H. Tian, S. Liu, J. Mei, and D. G. Chetwynd, "Optimal design of a 2-DOF pick-and-place parallel robot using dynamic performance indices and angular constraints," Mechanism \& Machine Theory, vol. 70, no. 6, pp. 246-253, 2013.

[54] S. Zhang and L. Kong, "Analysis on kinematics and dynamics performance indices for 6-PUS parallel robot mechanism," China Mechanical Engineering, vol. 15, no. 20, pp. 501-506, 2004.

[55] Z. Xie, F. Xie, X. Liu, J. Wang, and X. Shen, "Parameter optimization for the driving system of a 5 degrees of-freedom parallel machining robot with planar kinematic chains," Journal of Mechanisms and Robotics, vol. 11, no. 4, 2019.

[56] J. Wu, J. Wang, and Z. You, "An overview of dynamic parameter identification of robots," Robotics and ComputerIntegrated Manufacturing, vol. 26, no. 5, pp. 414-419, 2010. 